\title{
Diversidade de Arctiinae (Lepidoptera, Arctiidae) capturados com armadilha luminosa, em seis comunidades no Rio Grande do Sul, Brasil
}

\author{
José Augusto Teston ${ }^{1,2} \&$ Elio Corseuil ${ }^{3}$
}

'Pós-Graduação em Biociências, PUCRS. Av. Ipiranga, 6681. Caixa Postal 1429, 90619-900 Porto Alegre-RS, Brasil. Bolsista CNPq. ${ }^{2}$ Curso de Ciências Biológicas, Faculdade de Filosofia Ciências e Letras, UNICRUZ. Rua. Andrade Neves, 308, $98025-810$ Cruz Alta-RS, Brasil. Endereço eletrônico: jateston @ unicruz.edu.br

${ }^{3}$ Faculdade de Biociências, PUCRS. Av. Ipiranga, 6681. Caixa Postal 1429, 90619-900 Porto Alegre-RS, Brasil.

Endereço eletrônico: corseuil@pucrs.br

\begin{abstract}
Diversity of Arctiinae (Lepidoptera, Arctiidae) captured by light traps in six communities in Rio Grande do Sul State, Brazil. The Arctiinae fauna of Camaquã, Iraí, Lagoa Vermelha, Mostardas, Piratini, and São Pedro da Serra, were studied. The moths were caught by using light traps, once a month in the new moon phase, from January 1998 to December 1999. The species richness, abundance, constancy, as well as, the diversity and evenness indexes of Shannon and Brillouin, were used to evaluate the communities. Analysis of variance was used to evaluate the variation of individuals among months and localities. In order to estimate the species richness for each locality the nonparametrics statistics procedures Bootstrap, Chao 1, Chao 2, Jackknife 1, Jackknife 2 and Michaelis-Mentem have been used. A total of 9,800 specimens of Arctiinae belonging to 192 species, into 6 tribes were collected. The abundance and species richness were greater in 1998 than in 1999. The highest values of diversity indices in 1998 were found in Camaquã, Iraí and São Pedro da Serra; however in 1999 Iraí, Piratini and São Pedro da Serra were the districts with highest diversity. According to species richness estimators could be found more 34\% of species in Camaquã, 18\% in Iraí, 75\% in Lagoa Vermelha, $47 \%$ in Mostardas, $66 \%$ in Piratini and $43 \%$ in São Pedro da Serra.
\end{abstract}

KEYwORDs. Arctiidae; diversity; light trap; moths; Neotropical.

Resumo. Foram estudadas as faunas de Arctiinae em Camaquã, Iraí, Lagoa Vermelha, Mostardas, Piratini, e São Pedro da Serra. As mariposas foram capturadas por meio de armadilhas luminosas, uma vez por mês, na fase de lua nova, de janeiro de 1998 até dezembro de 1999. Na avaliação das comunidades, foram utilizados a riqueza de espécies, abundancia, constancia bem como, os índices de diversidade e uniformidade de Shannon e Brillouin. Para avaliar a variação do número de exemplares entre os meses e as localidades, foi realizada análise de variância. Na estimativa da riqueza de espécies para cada local, foram usados os procedimentos estatísticos não paramétricos "Bootstrap", "Chao 1", "Chao 2", "Jackknife 1", "Jackknife 2" e "Michaelis-Mentem". Foram capturados 9.800 exemplares de Arctiinae, pertencentes a 192 espécies e distribuídas em 6 tribos. A abundancia e riqueza de espécie, foram maiores em 1998 do que em 1999. Os maiores índices de diversidade em 1998 foram encontrados em Camaquã, Iraí e São Pedro da Serra; entretanto em 1999 Iraí, Piratini e São Pedro da Serra foram os locais de mais elevada diversidade. De acordo com os estimadores de riqueza de espécies podem ser encontradas mais 34\% de espécies em Camaquã, 18\% em Iraí, 75\% em Lagoa Vermelha, 47\% em Mostardas, $66 \%$ em Piratini e $43 \%$ em São Pedro da Serra.

Palavras-Chave. Arctiidae; armadilha luminosa; diversidade; mariposas; Neotropical.

Atualmente, um dos principais problemas enfrentados e conseqüentemente um assunto de muito interesse em todo o mundo é a perda da diversidade biológica pela degradação de ambientes e a respectiva destruição de suas populações naturais (HAYEK \& BUZAS 1997), tornando-se necessária a identificação e o registro de organismos o mais rapidamente possível (LANDAu et al. 1999).

Embora os insetos sejam o maior grupo de animais sobre o planeta, perfazendo mais da metade dos organismos vivos descritos, o conhecimento sobre os mesmos ainda é muito pequeno quando comparado a outros grupos. Seu uso em inventariamentos e estudos ambientais pode ser considerado insignificante (OLIVER \& BeATTIE 1996).

Nos últimos anos, devido ao interesse crescente pelo conhecimento dos recursos naturais no Brasil, a entomologia tornou-se uma ferramenta importante para a compreensão da diversidade biológica (FERREIRA et al. 1995). Isto deve-se ao fato de que os insetos são facilmente atraídos e capturados, e também por ocuparem os mais diversos hábitats.

Os lepidópteros, uma das principais ordens de insetos quanto à riqueza de espécies, são convenientes para estudos ambientais sobre a biodiversidade (INTACHAT \& WOIWOD 1999; LANDAU et al. 1999; KitChing et al. 2000), pois são importantes na dinâmica de ecossistemas, por que podem servir como bioindicadores, devido à sua ação como desfolhadores, decompositores, presas, hospedeiros e polinizadores (HAMmOND \& Miller 1998). Por serem em sua grande maioria fitófagos, podem servir como indicadores da qualidade da vegetação em diferentes tipos de hábitats (Kitching et al. 2000).

O uso de luz na captura de mariposas é muito difundido entre os pesquisadores, existindo vários estudos sobre modelos de armadilhas e fontes luminosas, demonstrando sua eficiência na coleta, para avaliar o número de indivíduos e 
espécies (FRY \& WARING 1996). O uso criterioso de armadilhas luminosas pode fornecer dados sobre a fauna local, que podem ser associados aos fatores bióticos e abióticos (LANDAU et al. 1999; Kitching et al. 2000). Alguns estudos, entre eles os de MARINONI \& DUTRA (1996) e BUTLER et al. (1999) alertam para fatores, tais como comprimento de onda emitido pela lâmpada, chuva, fase lunar e temperatura, que podem influenciar na sua adequação para a obtenção de amostragens para estudos sobre a ecologia de lepidópteros noturnos.

No Brasil, foram realizados estudos sobre a fauna de lepidópteros, incluindo Arctiidae, utilizando este método de captura, onde se destacam os de Dorval et al. (1995), Ferreira et al. (1995), LÜBECK et al. (1995), PEREIRA et al. (1995), MARINONI \& Dutra (1996) e CAMARgo (1999), analisando aspectos de dinâmica, diversidade e distribuição.

Atualmente, a subfamília Arctiinae (KITCHING \& RAwLINS 1999; JACOBSON \& Weller 2002) está representada mundialmente por 6.523 espécies, sendo que destas 4.761 somente na região Neotropical (HEPPNER 1991).

Segundo Hilty \& Merenlender (2000) Arctiidae, juntamente com Saturniidae e Sphingidae, estão entre os lepidópteros noturnos mais utilizados como bioindicadores no monitoramento de ecossistemas.

A avaliação das comunidades foi efetuada somente com a fauna de Arctiinae atraídas pela luz, já que algumas espécies diurnas não são capturadas.

$\mathrm{O}$ presente estudo teve como objetivos caracterizar e comparar as comunidades de Arctiinae em seis localidades do Rio Grande do Sul.

\section{MATERIALE MÉTODOS}

Foram realizadas coletas em seis locais do Rio Grande do Sul, no período de janeiro de 1998 a dezembro de 1999, uma vez por mês no período de lua nova, com duas armadilhas luminosas em cada local.

As coletas foram realizadas nos municípios de Iraí, Lagoa Vermelha, São Pedro da Serra, Camaquã, Mostardas e Piratini (Fig. 1) que, segundo Arend (1997), pertencem às zonas fisiográficas do Alto Uruguai, Campos de Cima da Serra, Encosta Inferior do Nordeste, Encosta do Sudeste, Litoral e Serra do Sudeste, respectivamente. A caracterização dos municípios, conforme a região fitoecológica segue LeiTE (2002) e Quadros \& Pillar (2002) e, quanto ao local de coleta, SPeChT (2001):

- Iraí, na Linha Baldim $\left(27^{\circ} 12^{\prime} \mathrm{S}, 53^{\circ} 13^{\prime} \mathrm{W}\right.$, altitude $\left.235 \mathrm{~m}\right)$, vegetação típica da Floresta Estacional Decidual, apresenta acentuada variação térmica com clima de duas estações, uma com médias acima de $20^{\circ} \mathrm{C}$ e outra abaixo dos $15^{\circ} \mathrm{C}$. Apresenta a vegetação típica bem conservada;

- Lagoa Vermelha, na Granja Canarinho (28 $18^{\circ}$ 'S, 51 ${ }^{\circ} 35^{\prime} \mathrm{W}$, altitude $830 \mathrm{~m}$ ), vegetação típica da Estepe Ombrófila, também com capões e florestas de galerias, e da Floresta Ombrófila Mista Montana, apresenta temperaturas significativamente mais baixas, com até oito meses de médias térmicas abaixo dos $15^{\circ} \mathrm{C}$. Ao redor do local encontram-se áreas de cultivos anuais;
- São Pedro da Serra, no Bairro Vila Nova $\left(29^{\circ} 25^{\prime} \mathrm{S}, 51^{\circ}\right.$ 28 'W, altitude $556 \mathrm{~m}$ ), vegetação típica da Floresta Ombrófila Mista e Floresta Estacional Semidecidual Moderada, caracteriza-se pela ocorrência de longo período frio com temperatura média inferior a $15^{\circ} \mathrm{C}$ e o período quente é curto com temperatura média igual ou superior a $20^{\circ} \mathrm{C}$. Devido ao relevo acidentado, a vegetação típica apresenta-se bem preservada.

- Camaquã, em Figueira Marcada ( $30^{\circ} 45^{\prime} \mathrm{S}, 51^{\circ} 52^{\prime} \mathrm{W}$, altitude $144 \mathrm{~m}$ ), vegetação típica da Floresta Estacional Semidecidual Moderada, apresentando clima do tipo quente úmido com médias térmicas, em geral, entre 15 e $20^{\circ} \mathrm{C}$. O local apresenta a vegetação típica bem conservada;

- Mostardas, no terceiro distrito Tunas ( $31^{\circ} 02^{\prime} \mathrm{S}, 50^{\circ} 57^{\prime} \mathrm{W}$, altitude $7 \mathrm{~m}$ ), vegetação típica de Formação Pioneira ou "Restinga". Apresenta áreas de pastagens e de atividade agrícola; também há alguns capões e nas margens dos canais e lagoas ocorrem juncos, gravatás e aguapés;

- Piratini, no Passo da Invernada ( $31^{\circ} 31^{\prime} \mathrm{S}, 53^{\circ} 05^{\prime} \mathrm{W}$, altitude $225 \mathrm{~m}$ ), vegetação típica da Estepe Estacional, com florestasde-galeria, apresenta temperaturas de verão bem elevadas enquanto que as de inverno, mais baixas, com médias inferiores a $15^{\circ} \mathrm{C}$. Apresenta vegetação conservada e com pouca alteração;

Foram utilizadas armadilhas luminosas INTRAL AL 012, modelo Pensilvânia (Frost 1957), equipadas com lâmpadas fluorescentes ultravioleta F15 T12 LN, cuja luz possui um comprimento de onda que varia de 290 a 450 e pico ao redor de 340 nanometros. Em cada armadilha foi fixado um tronco de cone plástico com maior diâmetro de $32 \mathrm{~cm}$ e menor de 16 , ao qual foi acoplado um balde plástico com capacidade de 3,5 litros. Cada aparelho foi instalado a uma altura de 3 metros do solo, ficando ligadas do anoitecer até o amanhecer do dia

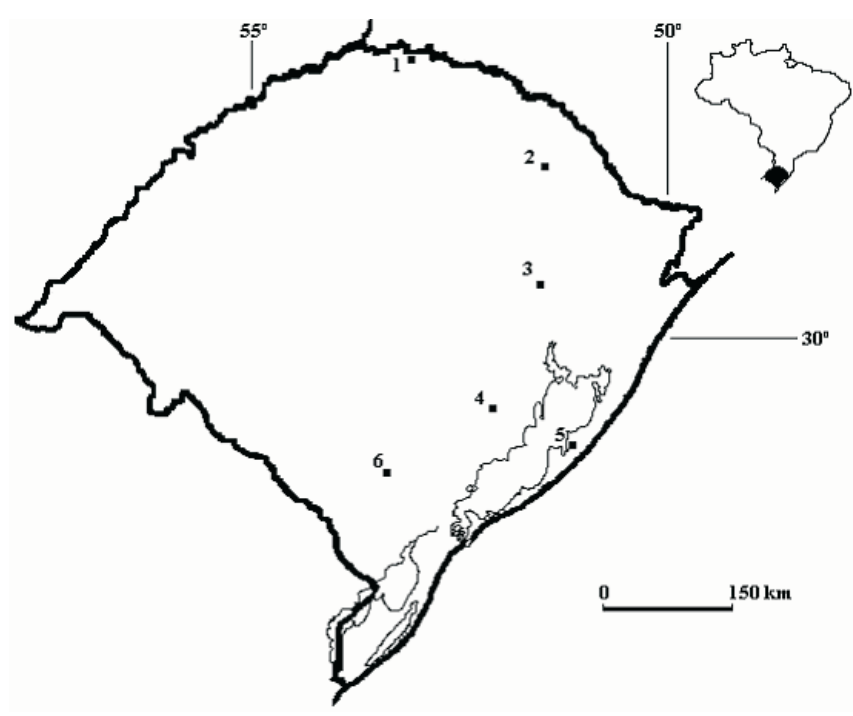

Fig. 1. Estado do Rio Grande do Sul, Brasil. Locais de coleta. 1, Iraí; 2 , Lagoa Vermelha; 3, São Pedro da Serra; 4, Camaquã; 5, Mostardas; 6, Piratini. 
seguinte. As armadilhas ficaram a uma distância aproximada de $800 \mathrm{~m}$, entre barreiras naturais, assegurando não ocorrer interferência de luminosidade entre as mesmas. A quantidade de amostras em cada local pode ser visualizada na Tabela V, sendo que as perdas de algumas coletas estão registradas nas Figs. 2 a 7 pela ausência de dados nos respectivos meses.

No Laboratório de Entomologia da Pontifícia Universidade Católica do Rio Grande do Sul (PUCRS), foi realizada a triagem do material e a montagem em alfinetes entomológicos, de exemplares representativos de cada espécie. O material testemunha encontra-se depositado nas coleções entomológicas do Museu de Ciências e Tecnologia da Pontifícia Universidade Católica do Rio Grande do Sul (MCTP) e particular do primeiro autor (CJAT).

A identificação das espécies foi realizada através de obras de referência, comparação com exemplares das coleções e auxílio de especialista, já referidos em listagens faunísticas anteriores (TESTON \& CoRseull 2002; 2003a, b).

O posicionamento sistemático adotado segue Kiтching \& RAwLINS (1999) e JACOBSON \& WeLleR (2002). A distribuição das espécies dentro dos gêneros segue WATSON \& Goodger (1986) para as tribos Arctiini, Callimorphini, Phaegopterini e Pericopini, e Hampson (1898) e Weller et al. (2000) para Ctenuchini e Euchromiini, com atualização de nomes genéricos por WATSON et al. (1995).

$\mathrm{Na}$ análise dos dados, calculou-se a constância das espécies em cada mês, classificando-as, segundo Bodenheimer referido por Silveira Neto et al. (1976), em constantes (presentes em mais de 50\% das coletas), acessórias (presentes entre $25-50 \%$ das coletas) e acidentais (presentes em menos de $25 \%$ das coletas). Também foram avaliadas as variações dos números de exemplares em cada local e mês, através de análise de variância, com os dados transformados em logaritmo base 10 de $(x+1)$, sendo as médias agrupadas pelo teste de Duncan ao nível de 5\%. Devido à acentuada diferença na abundância entre cada ano, os números de exemplares foram analisados como bifatoriais, por local e por mês, com duas repetições, para cada ano separadamente.

Foram utilizadas análises de regressão para avaliar a influência dos fatores climáticos sobre a ocorrência e abundância de espécies ao longo dos dois anos, no dia da coleta. Os parâmetros utilizados foram: temperatura e umidade relativa no dia de coleta, às 9,15 e 21 horas, precipitação pluviométrica no dia anterior e às 21 horas no dia de coleta. Os dados foram obtidos junto ao $8^{\circ}$ Distrito de Meteorologia, do Instituto Nacional de Meteorologia, em Porto Alegre. No caso de Mostardas, Piratini e São Pedro da Serra, municípios sem estações de medição, foram utilizados dados de estações meteorológicas mais próximas que foram as de Rio Grande, Bagé e Bento Gonçalves, respectivamente. Para Lagoa Vermelha, em 1998, foram utilizados dados de Caxias do Sul.

Para caracterizar as comunidades de Arctiinae em cada local, foram utilizados os parâmetros: riqueza de espécies (S); número de indivíduos $(\mathrm{N})$; índices de diversidade de Shannon $\left(H^{\prime}\right)$, cujos valores encontrados foram comparados pelo teste " $t$ " de Student, e de Brillouin ( $H$ ); uniformidade de Shannon ( $E^{\prime}$ ) e
Brillouin (E), referidos por MagurRAN (1988) e Krebs (1999), calculados através do programa de computador "Krebs Ecological Methodology for Windows" (BRzustowski 1997). As estimativas de riqueza de espécies foram realizadas com auxílio do programa de computador "Estimates Richness Estimator" (Colwell 2000), empregando os procedimento "Bootstrap", "Chao 1", "Chao 2", "Jackknife 1", "Jackknife 2" e "Michaelis-Mentem", utilizando 100 casualizações com abundância de classes igual a 10 (Colwell \& Coddington 1994). Demais cálculos e gráficos foram realizados utilizando o programa EXCEL da Microsoft.

\section{RESULTADOSEDISCUSSÃO}

Foram coletados 9.800 exemplares (Tabela I) dos quais, 7.773 em 1998 e 2.027 em 1999. Houve o registro de 192 espécies, com ocorrência de 178 em 1998 e 130 em 1999 . O total de espécies coletadas, neste estudo, corresponde a $59,1 \%$ das 325 registradas para o Estado (TESTON \& CORSEUIL 2002; 2003a, b). Foram encontrados representantes de todas as seis tribos ocorrentes na região Neotropical (Arctiini, Callimorphini, Ctenuchini, Euchromiini, Pericopini e Phaegopterini) e representantes dos gêneros Correbidia Hampson, 1898, Ochrodota Hampson, 1901 e Sphecosoma Butler, 1876 são registrados pela primeira vez para o Estado.

Em 1998, foram coletados aproximadamente quatro vezes mais exemplares do que em 1999, devido talvez aos fenômenos climáticos, "El Niño" e "La Niña" ocorrentes em 1998 e 1999, respectivamente. Segundo Lasmar (1997), o "El Niño" caracteriza-se pelo inverno com temperaturas amenas e a primavera com um índice pluviométrico muito alto. Já "La Niña" é, ao contrario, a passagem de frentes frias mais intensas e rápidas, desta forma não ocorrendo o acúmulo de muita chuva e, conseqüentemente, apresentando período de estiagem (GlantZ 1997). Deve-se observar que estes fenômenos climáticos começaram em meados de 1997, período anterior ao de início das coletas e que certamente influenciaram no ciclo biológico das espécies. Estas constatações quanto à relação entre número de exemplares e pluviometria também foram observadas por MARINONI \& DUTRA (1996) e SPECHT (2001). A mesma influência também pode ser atribuída às diferenças quanto ao número de espécies, pois em 1998 foram coletados 92,7\% do total registrado enquanto em 1999 foram constatados somente $67,7 \%$.

Os números mensais de espécies e exemplares, para cada local e totais durante os dois anos são apresentados nas Figs. 2 a 8 , onde se observa a grande diferença entre os anos e meses, quanto à abundância e riqueza de espécies, em todos os locais. Estas variações devem estar relacionadas a fenômenos climáticos, como já foram observadas por KITCHING et al. (2000) para Arctiidae na Austrália e também para lepidópteros diversos por СоОК \& GRAHAM (1996) na Inglaterra e por ButLer et al. (1999) nos Estados Unidos.

Ocorreram 91 espécies com até 10 exemplares (Tabela I) e que estiveram presentes no máximo em quatro locais, com abundâncias muito baixas, das quais 32 estão representadas 
Tabela I. Número de exemplares e constância ((A) = Acidentais, $(B)=$ Acessórias e $(C)=$ Constantes $)$ de Arctiinae capturados com armadilha luminosa em municípios do Rio Grande do Sul nos anos de 1998 e 1999. \# Espécies de ocorrência exclusiva em somente um local. * Gêneros registrados pela primeira vez para o Rio Grande do Sul.

\begin{tabular}{|c|c|c|c|c|c|c|c|c|c|c|c|c|c|}
\hline \multirow[t]{2}{*}{ Tribos / Espécies } & \multicolumn{2}{|c|}{ Camaquã } & \multicolumn{2}{|c|}{ Iraí } & \multicolumn{2}{|c|}{$\begin{array}{c}\text { Lagoa } \\
\text { Vermelha }\end{array}$} & \multicolumn{2}{|c|}{ Mostardas } & \multicolumn{2}{|c|}{ Piratini } & \multicolumn{2}{|c|}{$\begin{array}{c}\text { São Pedro } \\
\text { da Serra }\end{array}$} & \multirow[t]{2}{*}{ Total } \\
\hline & 1998 & 1999 & 1998 & 1999 & 1998 & 1999 & 1998 & 1999 & 1998 & 1999 & 1998 & 1999 & \\
\hline ARCTIINI & 9 & 5 & 21 & 9 & 33 & 31 & 4 & 20 & 10 & 39 & 28 & 5 & 214 \\
\hline 1. Hypercompe brasiliensis (Oberthür, 1881) & $1(\mathrm{~A})$ & & & & & & & & & & $1(\mathrm{~A})$ & & 2 \\
\hline Hypercompe cunigunda (Stoll, 1781) & & & $3(\mathrm{~A})$ & $1(\mathrm{~A})$ & & & & & & $1(\mathrm{~A})$ & & $1(\mathrm{~A})$ & 6 \\
\hline Hypercompe detecta (Oberthür, 1881) & $4(\mathrm{~A})$ & & $9(\mathrm{~B})$ & $3(\mathrm{~A})$ & & & $2(\mathrm{~A})$ & $2(\mathrm{~A})$ & & $10(\mathrm{~A})$ & $5(\mathrm{~A})$ & & 35 \\
\hline Hypercompe heterogena (Oberthür, 1881) & & $1(\mathrm{~A})$ & & & & & & & & & & & 1 \\
\hline Hypercompe indecisa (Walker, 1855) \# & & & & & & & & & $3(\mathrm{~A})$ & & & & 3 \\
\hline Hypercompe kinkelini (Burmeister, 1880) \# & & & & & & & & & & $1(\mathrm{~A})$ & & & 1 \\
\hline Hypercompe sp. & 1(A) & & & & $1(\mathrm{~A})$ & & & & & & & & 2 \\
\hline Isia intricata Walker, 1856 & & & $9($ в) & & $20($ в $)$ & $3($ в) & & & & & $22($ в) & & 54 \\
\hline Paracles albescens (Hampson, 1901) & $1(\mathrm{~A})$ & & & & $8(\mathrm{~A})$ & & & & & & & & 9 \\
\hline Paracles azollae (Berg, 1877) & & & & & & & $1(\mathrm{~A})$ & $17(\mathrm{c})$ & $1(\mathrm{~A})$ & $2(\mathrm{~A})$ & & & 21 \\
\hline Paracles bilinea (Schaus, 1901) & $1(\mathrm{~A})$ & & & $5($ в $)$ & & 27(в) & & & $1(\mathrm{~A})$ & $11(\mathrm{~B})$ & & $1(\mathrm{~A})$ & 46 \\
\hline Paracles costata (Burmeister, 1878) & & $2(\mathrm{~A})$ & & & & & & & & $5(\mathrm{~A})$ & & & 7 \\
\hline Paracles fusca (Walker, 1856) & & $1(\mathrm{~A})$ & & & & $1(\mathrm{~A})$ & & & & $2(\mathrm{~A})$ & & $3(\mathrm{~A})$ & 7 \\
\hline Paracles pallidivena (Schaus, 1904) \# & & $1(\mathrm{~A})$ & & & & & & & & & & & 1 \\
\hline Paracles variegata (Schaus, 1896) & $1(\mathrm{~A})$ & & & & 4(в) & & & & $5(\mathrm{~A})$ & $6(\mathrm{~A})$ & & & 16 \\
\hline Paracles vulpina (Hübner, [1825]) \# & & & & & & & $1(\mathrm{~A})$ & $1(\mathrm{~A})$ & & & & & 2 \\
\hline Paracles sp. \# & & & & & & & & & & $1(\mathrm{~A})$ & & & 1 \\
\hline CALLIMORPHINI & 1 & $\mathbf{0}$ & 4 & $\mathbf{0}$ & 1 & $\mathbf{0}$ & $\mathbf{0}$ & $\mathbf{0}$ & $\mathbf{0}$ & $\mathbf{0}$ & $\mathbf{0}$ & $\mathbf{0}$ & 6 \\
\hline 18. Utethesia ornatrix (Linnaeus, 1758) & $1(\mathrm{~A})$ & & $4($ в $)$ & & $1(\mathrm{~A})$ & & & & & & & & 6 \\
\hline CTENUCHINI & 1006 & 188 & 616 & 525 & 88 & 11 & 11 & 1 & 399 & 23 & 641 & 97 & 3606 \\
\hline 19. Aclytia heber (Cramer, 1780) & $127(\mathrm{c})$ & $7($ в ) & $90(\mathrm{c})$ & $44(\mathrm{c})$ & 17(в) & & & & $1(\mathrm{~A})$ & & $69($ в) & $1(\mathrm{~A})$ & 356 \\
\hline 20. Aclytia jonesi Rothschild, 1912 \# & & & $2(\mathrm{~A})$ & $2(\mathrm{~A})$ & & & & & & & & & 4 \\
\hline Aclytia terra Schaus, 1896 & $41($ в $)$ & $1(\mathrm{~A})$ & $63(c)$ & $39(\mathrm{c})$ & $6($ в $)$ & & & & $1(\mathrm{~A})$ & & $52(c)$ & 3(в) & 206 \\
\hline 22. Agyrta albisparsa Hampson, 1898 \# & $23($ в ) & & & & & & & & & & & & 23 \\
\hline 23. Argyroeides braco (Herrich-Schäffer, 1855 & $5)^{\#}$ & & $1(\mathrm{~A})$ & & & & & & & & & & 1 \\
\hline 24. Argyroeides flavipes Hampson, 1898 \# & & & $1(\mathrm{~A})$ & & & & & & & & & & 1 \\
\hline 25. Argyroeides sanguinea Schaus, 1896 \# & & & $5($ в ) & $1(\mathrm{~A})$ & & & & & & & & & 6 \\
\hline 26. Argyroeides vespina Schaus, 1901 \# & & & $1(\mathrm{~A})$ & & & & & & & & & & 1 \\
\hline 27. Argyroeides sp. \# & & & $1(\mathrm{~A})$ & & & & & & & & & & 1 \\
\hline 28. Callopepla grandis Rothschild, 1912 \# & & & & & & & & & $1(\mathrm{~A})$ & & & & 1 \\
\hline 29. Callopepla inachia (Schaus, 1892)\# & & & & & & & & & & & $1(\mathrm{~A})$ & & 1 \\
\hline 30. Correbidia sp. * \# & & & $1(\mathrm{~A})$ & & & & & & & & & & 1 \\
\hline Ctenucha divisa (Walker, 1856) & & & $2(\mathrm{~A})$ & $1(\mathrm{~A})$ & $4(\mathrm{~A})$ & & & & & & & & 7 \\
\hline Cyanopepla jucunda (Walker, 1854) \# & $1(\mathrm{~A})$ & & & & & & & & & & & & 1 \\
\hline Delphyre pyroperas Hampson, 1911 & & & $2(\mathrm{~A})$ & $8($ в $)$ & & & & & & & $2(\mathrm{~A})$ & $1(\mathrm{~A})$ & 13 \\
\hline Delphyre roseiceps Dognin, 1909 & $6($ в) & $1(\mathrm{~A})$ & $1(\mathrm{~A})$ & $1(\mathrm{~A})$ & & & $1(\mathrm{~A})$ & $1(\mathrm{~A})$ & & & $16($ в $)$ & $2(\mathrm{~A})$ & 29 \\
\hline Dinia aeagrus (Cramer, 1779) \# & & & $1(\mathrm{~A})$ & $1(\mathrm{~A})$ & & & & & & & & & 2 \\
\hline Diptilon aterea Schaus, 1901 \# & & & & & & & & & & & $1(\mathrm{~A})$ & & 1 \\
\hline 37. Diptilon doeri (Schaus, 1892) \# & & & $1(\mathrm{~A})$ & & & & & & & & & & 1 \\
\hline Diptilon halterata (Fabricius, 1775) \# & & & $6($ в ) & 13(в) & & & & & & & & & 19 \\
\hline Diptilon telamonaphorum Prittwitz, 1870 & & & $1(\mathrm{~A})$ & $10($ в $)$ & & & & & & & & & 11 \\
\hline Episcepsis endodasia Hampson, 1898 & $6($ в) & $1(\mathrm{~A})$ & & $1(\mathrm{~A})$ & & & & & & & $2(\mathrm{~A})$ & $1(\mathrm{~A})$ & 11 \\
\hline Eucereon apicalis (Walker, 1856) \# & & & & & & & & & & & $123(\mathrm{c})$ & & 123 \\
\hline Eucereon arenosum Butler, 1877 & $11(\mathrm{~B})$ & 2(A) & & & & & & & & & $9($ в $)$ & $1(\mathrm{~A})$ & 23 \\
\hline 43. Eucereon capsica (Schaus, 1896) & & & & $1(\mathrm{~A})$ & & & & & & & & $1(\mathrm{~A})$ & 2 \\
\hline Eucereon chalcodon Druce, 1893 & 14(в) & $3(\mathrm{~A})$ & & & & & $1(\mathrm{~A})$ & & & & $1(\mathrm{~A})$ & & 19 \\
\hline 45. Eucereon discolor (Walker, 1856) \# & & & & & & & & & & & $35($ в) & & 35 \\
\hline Eucereon ladas Schaus, 1892 & $2(\mathrm{~A})$ & & $1(\mathrm{~A})$ & $1(\mathrm{~A})$ & & $2(\mathrm{~A})$ & & & & & & & 6 \\
\hline 47. Eucereon quadricolor (Walker, 1855) & $10($ в $)$ & & $2(\mathrm{~A})$ & & & & & & & & $6($ в) & & 18 \\
\hline Eucereon marcata Schaus, 1901 & & & $4(\mathrm{~A})$ & $1(\mathrm{~A})$ & & & & & & & $1(\mathrm{~A})$ & & 6 \\
\hline 49. Eucereon rosa (Walker, 1854) & $132(\mathrm{c})$ & 11(в) & $10(\mathrm{c})$ & $9($ в $)$ & & & & & & $1(\mathrm{~A})$ & 20(в) & $6(\mathrm{~A})$ & 189 \\
\hline Eucereon striatum (Druce, 1889) & 13(в) & $1(\mathrm{~A})$ & & & & & & & & & $8($ в $)$ & $3(\mathrm{~B})$ & 25 \\
\hline Eucereon sp. \# & & & & & & & & & & & $15(\mathrm{~B})$ & $7(\mathrm{~B})$ & 22 \\
\hline Euceriodes pallada (Druce, 1906) & $6($ в) & $2(\mathrm{~A})$ & $1(\mathrm{~A})$ & $1(\mathrm{~A})$ & $1(\mathrm{~A})$ & & & & & & $2(\mathrm{~A})$ & & 13 \\
\hline 53. Euclera rubricincta (Burmeister, 1878) \# & & & & & & & & & $2(\mathrm{~A})$ & & & & 2 \\
\hline 54. Galethalea pica (Walker, 1855) & $2(\mathrm{~A})$ & & & & & & & & & & $1(\mathrm{~A})$ & & 3 \\
\hline 55. Hyaleucerea mundula (Berg, 1882) & & & & $32(\mathrm{~A})$ & & & & & & & $4(\mathrm{~A})$ & & 36 \\
\hline Nelphe mara (Kaye, 1914) & $11(\mathrm{~A})$ & & $3($ в ) & $30(\mathrm{c})$ & $4($ в $)$ & & & & & & 14(в) & & 62 \\
\hline Philoros affinis (Rothschild, 1912) & $585(\mathrm{c})$ & $159(\mathrm{c}) 4$ & $402(\mathrm{C})$ & $317(\mathrm{c})$ & $55(\mathrm{c})$ & $8(\mathrm{~A})$ & $9($ в $)$ & & 394(c) & $22($ в $) 2$ & $250(\mathrm{c})$ & $70(\mathrm{c})$ & 2271 \\
\hline 58. Pseudohyaleucerea vulnerata Butler, 1875 & $512($ в) & & $7($ в ) & $3(\mathrm{~A})$ & $1(\mathrm{~A})$ & & & & & & $2(\mathrm{~A})$ & $1(\mathrm{~A})$ & 26 \\
\hline 59. Pseudosphex noverca Schaus, 1901 & & & & $1(\mathrm{~A})$ & & & & & & & $1(\mathrm{~A})$ & & 2 \\
\hline 60. Sciopsyche tropica (Walker, 1854) \# & & & $6($ в $)$ & & & & & & & & & & 6 \\
\hline Theages leucophaea Walker, 1855 \# & & & & & & & & & & & $3(\mathrm{~A})$ & & 3 \\
\hline 62. Tipulodes ima Boisduval, 1832 & $4(\mathrm{~A})$ & & $1(\mathrm{~A})$ & $8(\mathrm{~A})$ & & $1(\mathrm{~A})$ & & & & & $3(\mathrm{~A})$ & & 17 \\
\hline
\end{tabular}


Tabela I. Cont.

\begin{tabular}{|c|c|c|c|c|c|c|c|c|c|c|c|c|c|}
\hline \multirow[t]{2}{*}{ Tribos / Espécies } & \multicolumn{2}{|c|}{ Camaquã } & \multicolumn{2}{|c|}{ Iraí } & \multicolumn{2}{|c|}{$\begin{array}{c}\text { Lagoa } \\
\text { Vermelha }\end{array}$} & \multicolumn{2}{|c|}{ Mostardas } & \multicolumn{2}{|c|}{ Piratini } & \multicolumn{2}{|c|}{$\begin{array}{l}\text { São Pedro } \\
\text { da Serra }\end{array}$} & \multirow[t]{2}{*}{ Total } \\
\hline & 1998 & 1999 & 1998 & 1999 & 1998 & 1999 & 1998 & 1999 & 1998 & 1999 & 1998 & 1999 & \\
\hline EUCHROMIINI & 540 & 55 & 277 & 332 & 51 & 10 & 1 & $\mathbf{0}$ & 60 & 15 & 636 & 110 & 2087 \\
\hline $\begin{array}{l}\text { 63. Aristodaema hanga (Herrich-Schäffer, } \\
[1854])\end{array}$ & $1(\mathrm{~A})$ & & $2(\mathrm{~A})$ & $3(\mathrm{~B})$ & $2(\mathrm{~A})$ & & & & & & & $4(\mathrm{~B})$ & 12 \\
\hline 64. Cosmosoma auge (Linnaeus, 1767) & $75(\mathrm{c})$ & $3(\mathrm{~A})$ & $1(\mathrm{~A})$ & $1(\mathrm{~A})$ & & & & & & & 48(в) & $4($ в) & 132 \\
\hline 65. Cosmosoma centrale (Walker, 1854) & $3($ в) & $3(\mathrm{~A})$ & $35(\mathrm{c})$ & 130(в) & 13(в) & $5(\mathrm{~A})$ & $1(\mathrm{~A})$ & & $1(\mathrm{~A})$ & & $62(\mathrm{c})$ & $28(\mathrm{c})$ & 281 \\
\hline 66. Cosmosoma leuconoton Hampson, 1898 & $5(\mathrm{~A})$ & & $4(\mathrm{~B})$ & $3(\mathrm{~B})$ & $1(\mathrm{~A})$ & & & & & & & & 13 \\
\hline 67. Cosmosoma plutona Schaus, 1894 \# & & & $1(\mathrm{~A})$ & & & & & & & & & & 1 \\
\hline 68. Cosmosoma telephus (Walker, 1854) & & & $2(\mathrm{~A})$ & $2(\mathrm{~A})$ & & & & & & & & $2(\mathrm{~A})$ & 6 \\
\hline 69. Cosmosoma teuthras (Walker, 1854) \# & & & $6(\mathrm{~B})$ & & & & & & & & & & 6 \\
\hline 70. Cosmosoma sp. \# & 1(A) & & & & & & & & & & & & 1 \\
\hline 71. Dycladia lucetius (Stoll, 1781) & 26(в) & 3(в) & $45(\mathrm{c})$ & $91(\mathrm{c})$ & $4(\mathrm{~A})$ & & & & 1(A) & & $3(\mathrm{~A})$ & $2(\mathrm{~A})$ & 175 \\
\hline 72. Eurota helena (Herrich-Schäffer, 1854) & 2(A) & $1(\mathrm{~A})$ & $12(\mathrm{~B})$ & $12(\mathrm{~B})$ & $4(\mathrm{~B})$ & $1(\mathrm{~A})$ & & & $3(\mathrm{~A})$ & & $7(\mathrm{~A})$ & $1(\mathrm{~A})$ & 43 \\
\hline 73. Eurota herricki Butler, 1876 & $3(\mathrm{~B})$ & & & & $7(\mathrm{~B})$ & $4(\mathrm{~B})$ & & & $45(\mathrm{c})$ & 11(B) & 1(A) & & 71 \\
\hline 74. Eurota stictibasis Hampson, 1898 \# & & & & & $1(\mathrm{~A})$ & & & & & & & & 1 \\
\hline 75. Holophaea erharda Schaus, 1927 & 29(в) & 1(A) & 1(A) & $1(\mathrm{~A})$ & & & & & $1(\mathrm{~A})$ & & 73(c) & 14(в) & 120 \\
\hline $\begin{array}{l}\text { 76. Horama panthalon viridifusa } \\
\text { (Schaus, 1894) }\end{array}$ & $1(\mathrm{~A})$ & $1(\mathrm{~A})$ & $11($ в) & $2(\mathrm{~A})$ & & & & & & & & & 15 \\
\hline 77. Ichoria chalcomedusa Druce, 1893 & & & $5(\mathrm{~A})$ & $2(\mathrm{~A})$ & & & & & & & $1(\mathrm{~A})$ & & 8 \\
\hline 78. Ichoria tricincta (Herrich-Schäffer, 1855) & & & & & $1(\mathrm{~A})$ & & & & & & $11(\mathrm{~B})$ & $3(\mathrm{~A})$ & 15 \\
\hline 79. Lepidoneiva erubescens (Butler, 1876) & & & 1(A) & & & & & & & & $1(\mathrm{~A})$ & & 2 \\
\hline 80. Macrocneme cupreipennis Walker, 1856 & 146(c) & 18(в) & $53(\mathrm{c})$ & $47(\mathrm{c})$ & & & & & $6(\mathrm{~A})$ & $3(\mathrm{~A})$ & 36(в) & $6($ в) & 315 \\
\hline 81. Macrocneme leucostigma (Perty, 1833) & $42(\mathrm{~B})$ & $9(\mathrm{~A})$ & $3(\mathrm{~A})$ & & & & & & & & & & 54 \\
\hline 82. Macrocneme pelotas Dietz, 1994 \# & $1(\mathrm{~A})$ & & & & & & & & & & & & 1 \\
\hline 83. Macrocneme sp. & $1(\mathrm{~A})$ & & $12(\mathrm{~A})$ & & & & & & & & & & 13 \\
\hline 84. Mallodeta clavata (Walker, 1854) & 2(A) & 1(A) & & & & & & & & & $2(\mathrm{~A})$ & & 5 \\
\hline 85. Mesothen desperata (Walker, 1856) & $1(\mathrm{~A})$ & & $10($ в $)$ & $3($ в) & & & & & & & $15(\mathrm{c})$ & $5($ в) & 34 \\
\hline 86. Mirandisca harpalyce (Schaus, 1892) & $16(\mathrm{~B})$ & $2(\mathrm{~A})$ & $2(\mathrm{~A})$ & & & & & & & & $12(\mathrm{~B})$ & $2(\mathrm{~A})$ & 34 \\
\hline 87. Neotrichura nigripes (Heylaerts, 1890) & & & $6($ в) & $4(\mathrm{~A})$ & & & & & & & & $1(\mathrm{~A})$ & 11 \\
\hline 88. Nyridela chalciope (Hübner, [1831]) \# & 1(A) & & & & & & & & & & & & 1 \\
\hline 89. Phoenicoprocta analis Schrottky, 1909 & & & $32(\mathrm{c})$ & $14(\mathrm{~B})$ & $1(\mathrm{~A})$ & & & & & & $2(\mathrm{~A})$ & $2(\mathrm{~A})$ & 51 \\
\hline 90. Phoenicoprocta rubiventer Hampson, 1898 & & & & & & & & & $2(\mathrm{~A})$ & & & & 2 \\
\hline 91. Phoenicoprocta teda (Walker, 1854) & $1(\mathrm{~A})$ & & $1(\mathrm{~A})$ & $1(\mathrm{~A})$ & & & & & & & & & 3 \\
\hline 92. Poliopastea indistincta (Butler, 1876) & $35($ в) & $1(\mathrm{~A})$ & $2(\mathrm{~A})$ & $1(\mathrm{~A})$ & & & & & & & 212(c) & $15(\mathrm{c})$ & 266 \\
\hline 93. Psilopleura sanguipuncta Hampson, 1898 & $1(\mathrm{~A})$ & & & & 11(A) & & & & & & & & 12 \\
\hline 94. Rhynchopyga meisteri (Berg, 1883) & $108(\mathrm{c})$ & $10($ в $)$ & $12(\mathrm{~B})$ & $8($ в $)$ & $6(\mathrm{~A})$ & & & & $1(\mathrm{~A})$ & $1(\mathrm{~A}) 1$ & 104(c) & $20(c)$ & 270 \\
\hline 95. Saurita bipuncta Hampson, 1898 & $25($ в) & $2(\mathrm{~A})$ & $3(\mathrm{~A})$ & & & & & & & & $44($ в) & $1(\mathrm{~A})$ & 75 \\
\hline 96. Saurita cassandra (Linnaeus, 1758) & $14(\mathrm{~B})$ & & $15(\mathrm{~B})$ & $7($ в $)$ & & & & & & & $1(\mathrm{~A})$ & & 37 \\
\hline 97. Sphecosoma sp. *\# & & & & & & & & & & & $1(\mathrm{~A})$ & & 1 \\
\hline PERICOPINI & 239 & 17 & 272 & 22 & 29 & 5 & 1 & $\mathbf{0}$ & 10 & 4 & 173 & 3 & 775 \\
\hline 98. Calodesma collaris (Drury, 1782) & & & $33(\mathrm{c})$ & $2(\mathrm{~A})$ & & & & & & & & & 35 \\
\hline 99. Calodesma dioptis (R. Felder, 1874) \# & & & $1(\mathrm{~A})$ & & & & & & & & & & 1 \\
\hline 100. Dysschema amphissa (Geyer, 1832) & $5($ в) & 1(A) & 1(A) & & & & & & & & 14(в) & & 21 \\
\hline 101. Dysschema hilarina (Weymer, 1914) & $11(\mathrm{~B})$ & $1(\mathrm{~A})$ & & & $20(\mathrm{c})$ & $4(\mathrm{~A})$ & & & 6(в) & $3(\mathrm{~A})$ & 2(A) & & 47 \\
\hline 102. Dysschema hypoxantha Hübner, $1818^{\#}$ & & & $7(\mathrm{~A})$ & & & & & & & & & & 1 \\
\hline 103. Dysschema neda (Klug, 1836) & $46(c)$ & & & & $3(\mathrm{~B})$ & & & & & & $61(\mathrm{c})$ & $1(\mathrm{~A})$ & 111 \\
\hline $\begin{array}{l}\text { 104. Dysschema picta (Guérin-Méneville, } \\
{[1844] \text { ) }}\end{array}$ & $9($ в) & $3(\mathrm{~A})$ & $1(\mathrm{~A})$ & & & & & & & & $4($ в) & & 17 \\
\hline 105. Dysschema sacrifica (Hübner, [1831]) & 3(в) & $1(\mathrm{~A})$ & 28(в) & & $5($ в) & & 1(A) & & 1(A) & & $16(\mathrm{~A})$ & & 55 \\
\hline 106. Dysschema trapeziata (Walker, $[1865]$ ) \# & & & & & 1(A) & $1(\mathrm{~A})$ & & & & & & & 2 \\
\hline 107. Euchlaenidia transcisa (Walker, 1854) & 139(c) & $5($ в) & 152(c) & $11($ в) & & & & & 3(A) & & 73(в) & $2(\mathrm{~A})$ & 385 \\
\hline 108. Hyalurga syma (Walker, 1854$)$ \# & & & 1(A) & & & & & & & & & & 1 \\
\hline 109. Phaloe cruenta (Hübner, 1823) & $26($ в $)$ & $6(\mathrm{~A})$ & $48(\mathrm{c})$ & $9($ в $)$ & & & & & & $1(\mathrm{~A})$ & $3($ в) & & 93 \\
\hline PHAEGOPTERINI & 973 & 104 & 459 & 109 & 326 & 86 & 61 & 7 & 79 & 53 & 714 & 141 & 3112 \\
\hline 110. Agaraea se & $41(\mathrm{~B})$ & 11(в) & $3($ в) & $3($ в) & & & & & $1(\mathrm{~A})$ & & 20 (в) & $15(\mathrm{c})$ & 94 \\
\hline 111. Aemilia asignata Hampson, 1901 & $1(\mathrm{~A})$ & & $5(\mathrm{~A})$ & & & & & & & & $1(\mathrm{~A})$ & $3(\mathrm{~A})$ & 10 \\
\hline 112. Amastus sp. 1 \# & & & & & & $1(\mathrm{~A})$ & & & & & & & 1 \\
\hline 113. Amastus sp. $2 \#$ & & & & & & 1(A) & & & & & & & 1 \\
\hline 114. Amastus sp. $3^{\#}$ & & & & & $8($ в ) & 1(A) & & & & & & & 9 \\
\hline 115. Amaxia corata $\mathrm{Sc}$ & $8($ в) & $1(\mathrm{~A})$ & & & & & & & & & & & 9 \\
\hline 116. Amaxia hebe Schaus, 1892 & $4($ в) & & & & & & & & & & 2(A) & & 6 \\
\hline 117. Ammalo helops (Cramer, 1775) & $2(\mathrm{~A})$ & & $2(\mathrm{~A})$ & & & & $2(\mathrm{~A})$ & & & & 21(A) & & 27 \\
\hline 118. Baritius acuminata (Walker, 1856) & $9(\mathrm{в})$ & 1(A) & 28(в) & 31(в) & $5($ в) & & & & 1(A) & & 20 (в) & 1(A) & 96 \\
\hline 119. Bertholdia soror Dyar, 1901 & $63(\mathrm{c})$ & $13(\mathrm{~B})$ & $92(\mathrm{c})$ & $13(\mathrm{~B})$ & $27(\mathrm{c})$ & $2(\mathrm{~A})$ & & & $4(\mathrm{~A})$ & $2(\mathrm{~A})$ & $69(\mathrm{c})$ & $17(\mathrm{~B})$ & 302 \\
\hline $\begin{array}{l}\text { 120. Bertholdia specularis (Herrich-Schäffer, } \\
{[1853] \text { ) }}\end{array}$ & $2(\mathrm{~A})$ & & 9 (в) & $1(\mathrm{~A})$ & & & & & & & $2(\mathrm{~A})$ & & 14 \\
\hline 121. Biturix rectilinea (Burmeister, 1878) & $5($ в) & $2(\mathrm{~A})$ & & & 1(A) & & & & & & 7(в) & $5(\mathrm{~A})$ & 20 \\
\hline 122. Carales astur (Cramer, 1777) & $6(\mathrm{~B})$ & $1(\mathrm{~A})$ & $3($ в) & $1(\mathrm{~A})$ & & $1(\mathrm{~A})$ & & & & & & $2(\mathrm{~A})$ & 14 \\
\hline 123. Demolis albicostata Hampson, 1901 & $8($ в) & & $2(\mathrm{~A})$ & & & & & & $2(\mathrm{~A})$ & & & & 12 \\
\hline 124. Echeta divisa (Herrich-Schäffer, [1855]) & 1(A) & & & & 1(A) & & & & & & & & 2 \\
\hline
\end{tabular}


Tabela I. Cont.

\begin{tabular}{|c|c|c|c|c|c|c|c|c|c|c|c|c|c|}
\hline \multirow[t]{2}{*}{ Tribos / Espécies } & \multicolumn{2}{|c|}{ Camaquã } & \multicolumn{2}{|c|}{ Iraí } & \multicolumn{2}{|c|}{$\begin{array}{c}\text { Lagoa } \\
\text { Vermelha }\end{array}$} & \multicolumn{2}{|c|}{ Mostardas } & \multicolumn{2}{|c|}{ Piratini } & \multicolumn{2}{|c|}{$\begin{array}{c}\text { São Pedro } \\
\text { da Serra }\end{array}$} & Total \\
\hline & 1998 & 1999 & 1998 & 1999 & 1998 & 1999 & 1998 & 1999 & 1998 & 81999 & 1998 & 1999 & \\
\hline 125. Elysius jonesi Rothschild, 1910 & $2(\mathrm{~A})$ & $3(\mathrm{~A})$ & & & 1(A) & $3($ в) & & & 4(A) & $1(\mathrm{~A})$ & $9($ в) & 2(A) & 25 \\
\hline 126. Elysius pyrosticta Hampson, 1905 & $20(\mathrm{c})$ & $2(\mathrm{~A})$ & 11(в) & $2(\mathrm{~A})$ & 1(A) & & & & & & 14(в) & 2(A) & 52 \\
\hline 127. Euchaetes rizoma (Schaus, 1896) \# & & & & & & $2(\mathrm{~A})$ & & & & & & & 2 \\
\hline 128. Eupseudosoma involuta (Sepp, [1855]) \# & & & $5(\mathrm{~B})$ & & & & & & & & & & 5 \\
\hline 129. Halysidota brasiliensis Rothschild, $1909^{\#}$ & & & $5(\mathrm{~A})$ & & & & & & & & & & 5 \\
\hline 130. Halysidota pearsoni Watson, 1980 & 11(в) & 4(в) & $8($ в) & 1(A) & & $1(\mathrm{~A})$ & & & $2(\mathrm{~A})$ & & $42($ в) & $24(\mathrm{c})$ & 93 \\
\hline 131. Halysidota striata Jones, $1908^{\#}$ & & & & & $5(\mathrm{~A})$ & $9(\mathrm{~A})$ & & & & & & & 14 \\
\hline 132. Halysidota sp. 1 & 3(в) & & & & & & & & & & 1(A) & & 4 \\
\hline 133. Halysidota sp. 2 \# & & & 1(A) & & & & & & & & & & 1 \\
\hline 134. Halysidota sp. $3^{\#}$ & & & 1(A) & & & & & & & & & & $\mathbf{1}$ \\
\hline 135. Hemihyalea diminuta (Walker, 1855) & 1(A) & & & & & & & & 14(A) & 1(A) & & & 16 \\
\hline 136. Hyalarctia sericera Schaus, 1901 & & & 1(A) & $1(\mathrm{~A})$ & 1(A) & 1(A) & & & & & & & 4 \\
\hline 137. Hyperthaema albipuncta Schaus, 1901 & & & & & $3(\mathrm{~A})$ & $2(\mathrm{~A})$ & & & $5(\mathrm{c})$ & $3(\mathrm{~A})$ & & & 13 \\
\hline 138. Hyperthaema sanguineata (Walker, [1865])\# & & & $2(\mathrm{~A})$ & 2(A) & & & & & & & & & 4 \\
\hline 139. Hyperthaema signatus (Walker, 1862) & 2(A) & 1(A) & & & & & & & $3(\mathrm{~A})$ & 1(A) & & & 7 \\
\hline 140. Hyperthaema sp. \# & & & & & 1(A) & $1(\mathrm{~A})$ & & & & & & & 2 \\
\hline 141. Hypidalia enervis (Sch & 12(в) & 2(A) & & & $30(\mathrm{c})$ & $6($ в) & 1(A) & & & & & & 51 \\
\hline 142. Idalus agastus Dyar, 1910 & $20(\mathrm{~B})$ & $5(\mathrm{~B})$ & $5(\mathrm{~B})$ & 1(A) & 6(в) & & 12(в) & 4(в) & $10(\mathrm{~B})$ & 1(A) & $1(\mathrm{~A})$ & & 65 \\
\hline 143. Idalus citrina Druce, $1890^{\#}$ & $3(\mathrm{~A})$ & 1(A) & & & & & & & & & & & 4 \\
\hline 144. Idalus noiva (Jones, 1914) \# & $1(\mathrm{~A})$ & & & & & & & & & & & & 1 \\
\hline 145. Idalus v & & & $1(\mathrm{~A})$ & & & & & & & & & & 1 \\
\hline 146. Ischnocampa sp. & & & $8(\mathrm{~B})$ & & $3(\mathrm{~A})$ & & & & & & $3(\mathrm{~A})$ & & 14 \\
\hline 147. Leucanopsis acuta (Hampson, 1901) & 1(A) & 1(A) & $4($ в) & & $3(\mathrm{~A})$ & & & & & & $1(\mathrm{~A})$ & & 10 \\
\hline 148. Leucanopsis calvona & & & 3(A) & & & & & & & & & & 3 \\
\hline $\begin{array}{l}\text { 149. Leucanopsis leucanina (R. Felder \& } \\
\text { Rogenhofer, 1874) }\end{array}$ & $13(\mathrm{c})$ & $1(\mathrm{~A})$ & $7($ в) & $1(\mathrm{~A})$ & $86(c)$ & $2(\mathrm{~A})$ & $37(\mathrm{c})$ & $2(\mathrm{~A})$ & $5($ в) & 3(в) & $42(\mathrm{c})$ & $1(\mathrm{~A})$ & 200 \\
\hline 150. Leucanopsis lineata (Schaus, 1894) \# & $7($ в) & & & & & & & & & & & & 7 \\
\hline 151. Leucanopsis quanta & & & & & $5(\mathrm{~B})$ & & & & & & & & 5 \\
\hline 152. Leucanopsis oruba & $8($ в $)$ & & $3(\mathrm{~A})$ & & & & & & & & $4($ в) & & 15 \\
\hline 153. Leucanopsis umbrosa (Hampson, 1901) \# & & & $11(\mathrm{c})$ & $2(\mathrm{~A})$ & & & & & & & & & 13 \\
\hline 154. Leucanopsis sp. 1 & $5($ в) & & $5(\mathrm{~A})$ & & 7(в) & 1(A) & & & $2(\mathrm{~A})$ & 1(A) & $9($ в) & & 30 \\
\hline 155. Leucanopsis sp. 2 & $8($ в) & & 11(в) & & & & & & & & $3(\mathrm{~A})$ & 1(A) & 23 \\
\hline 156. Lеисс & $4(\mathrm{~A})$ & & & & & & & & & & & & 4 \\
\hline 157. Leucanopsis sp. 4 & $10(\mathrm{~A})$ & & & & $2(\mathrm{~A})$ & 2(A) & & & & & $2(\mathrm{~A})$ & & 16 \\
\hline 158. Lophocampa arpi (Dognin, 1923) \# & & & & & & & & & & $10(\mathrm{~B})$ & & & 10 \\
\hline 159. Lophocampa catenulata (Hübner, [1812]) & $34(\mathrm{c})$ & $5(\mathrm{~A})$ & 8(в) & 1(A) & & & 7(A) & 1(A) & $7($ в) & 1(A) & $8(\mathrm{~A})$ & 1(A) & 73 \\
\hline 160. Lophocampa citrina (Sepp, [1843]) \# & $1(\mathrm{~A})$ & & & & & & & & & & & & 1 \\
\hline 161. Lophocampa texta $(\mathrm{H}$ & ]) $64(\mathrm{c})$ & 4(в) & $29(\mathrm{c})$ & & $5($ в) & 1(A) & & & $2(\mathrm{~A})$ & & $27(\mathrm{c})$ & & 132 \\
\hline 162. Machadoia xanthost & & & & & $82($ в) & $37(\mathrm{~B})$ & & & $5(\mathrm{~A})$ & $12(\mathrm{~A})$ & $237(\mathrm{c})$ & 53(в) & 426 \\
\hline 163. Mazaeras conferta & & & 1(A) & & & 1(A) & & & & & $5($ в) & $4(\mathrm{~A})$ & 11 \\
\hline 164. Mazaeras janeira & 1(A) & & & & & 1(A) & & & & & & & 2 \\
\hline 165. Melese castrena Sch & $28(\mathrm{c})$ & $3(\mathrm{~A})$ & $2(\mathrm{~A})$ & $2(\mathrm{~A})$ & & & & & & & $2(\mathrm{~A})$ & & 37 \\
\hline 166. Melese chozeba (Dru & 153(c) & $18(\mathrm{~B})$ & $12(\mathrm{c})$ & $1(\mathrm{~A})$ & & & & & & & $6($ в) & 1(A) & 191 \\
\hline 167. Munona iridescens $S$ & & & 4(в) & 7 (в) & & & & & & & $3(\mathrm{~A})$ & & 14 \\
\hline 168. Neidalia dulcicula Schaus, 1929 \# & & & & & & 4(в) & & & & & & & 4 \\
\hline 169. Neonerita dorsipuncta Hampson, 1901 & $5($ в) & 2(A) & 4(в) & 2(A) & & & & & & & 1(A) & & 14 \\
\hline 170. Neritos repanda Walker, 1855 & $43(\mathrm{c})$ & $5($ в) & $29(\mathrm{c})$ & $6($ в $)$ & $1(\mathrm{~A})$ & & & & $3(\mathrm{~A})$ & & $8($ в) & & 95 \\
\hline 171. Ochrodota sp. * \# & & & $5(\mathrm{~B})$ & & & & & & & & & & 5 \\
\hline 172. Opharus basalis Walker, 1856 & $2(\mathrm{~A})$ & & $2(\mathrm{~A})$ & & & & & & & & $1(\mathrm{~A})$ & & 5 \\
\hline 173. Opharus bimaculata (Dewitz, 1877) \# & & & & & & & & & & & 1(A) & & 1 \\
\hline 174. Opharus intermedia Rothschild, 1909 & & & & & & & & & & 11(в) & $1(\mathrm{~A})$ & & 12 \\
\hline 175. Opharus procroides V & $6(\mathrm{~A})$ & 1(A) & $36(\mathrm{c})$ & $8($ в) & 1(A) & & & & & & $85(\mathrm{c})$ & 1(A) & 138 \\
\hline 176. Opharus $r$ & $2(\mathrm{~A})$ & 1(A) & & & 1(A) & & & & & & & & 4 \\
\hline 177. Ormetica chrysomelas (Walker, 1856) & 103(c) & 6(в) & $22(\mathrm{c})$ & $6($ в) & & 1(A) & & & & & 14(в) & $3(\mathrm{~A})$ & 155 \\
\hline 178. Pareuchaetes aurata (Butler, 1875) & $117(\mathrm{c})$ & 2(A) & $34(\mathrm{c})$ & $3(\mathrm{~A})$ & 1(A) & & & & & & $17($ в) & 1(A) & 175 \\
\hline 179. Pelochyta cinerea (Walker, 1855) & $32(\mathrm{c})$ & $3($ в) & 6(в) & $5(\mathrm{~A})$ & 19 (в) & $2(\mathrm{~A})$ & & & $2(\mathrm{~A})$ & & 17(в) & 1(A) & 87 \\
\hline 180. Pelochyta sp. \# & & & $4($ в) & & & & & & & & & & \\
\hline 181. Phaegoptera albimacula (Jones, 1908) & & & & & 1(A) & & & & & & $1(\mathrm{~A})$ & & 2 \\
\hline 182. Phaegoptera chorima Schaus, $1896^{\#}$ & & & & & & & & & & & $1(\mathrm{~A})$ & & 1 \\
\hline $\begin{array}{l}\text { 183. Phaegoptera flavostrigata Herrich-Schäffer, } \\
\text { [1855] }\end{array}$ & $5(\mathrm{~A})$ & & & & & & & & $2(\mathrm{~A})$ & $2(\mathrm{~A})$ & & 2(A) & 11 \\
\hline 184. Rhipha flavoplagiata (Rothschild, 1911) & $10(\mathrm{~A})$ & $1(\mathrm{~A})$ & & & $1(\mathrm{~A})$ & & & & & & & & 12 \\
\hline 185. Rhipha subflammans (Rothschild, 1909) \# & & & & & & & & & & $2(\mathrm{~A})$ & & & 2 \\
\hline 186. Romиaldia elongata (R. Felder, 1874) & & & 1(A) & 1(A) & 13(A) & $2(\mathrm{~A})$ & & & & & & & 17 \\
\hline 187. Sychesia $d$ & $1(\mathrm{~A})$ & & & & & & & & & & & & 1 \\
\hline 188. Symphlebia catenata (Schaus, 1905) & $42(\mathrm{c})$ & 1(A) & 11(в) & $3(\mathrm{~A})$ & 1(A) & & $2(\mathrm{~A})$ & & $2(\mathrm{~A})$ & & $2(\mathrm{~A})$ & & 64 \\
\hline 189. Tessella sertata (Berg, 1882) & $11(\mathrm{~B})$ & & $5(\mathrm{~B})$ & $3($ в) & & & & & $2(\mathrm{~A})$ & & $3($ в) & & 24 \\
\hline 190. Tessellarctia semivaria (Walker, 1856) & 24(c) & $3(\mathrm{~A})$ & & & 3(в) & 1(A) & & & 1(A) & & & $1(\mathrm{~A})$ & 33 \\
\hline 191. Tessellota trifasciata (Burmeister, 1878) \# & & & & & & & & & & $2(\mathrm{~A})$ & & & 2 \\
\hline 192. Viviennea dolens (Druce, 1904) & $8($ в) & & 8(в) & $2(\mathrm{~A})$ & 1(A) & & & & & & 1(A) & & 20 \\
\hline TOTAIS & 2768 & 369 & 1649 & 997 & 528 & 143 & 78 & 28 & 558 & 134 & 2192 & 356 & 9800 \\
\hline
\end{tabular}




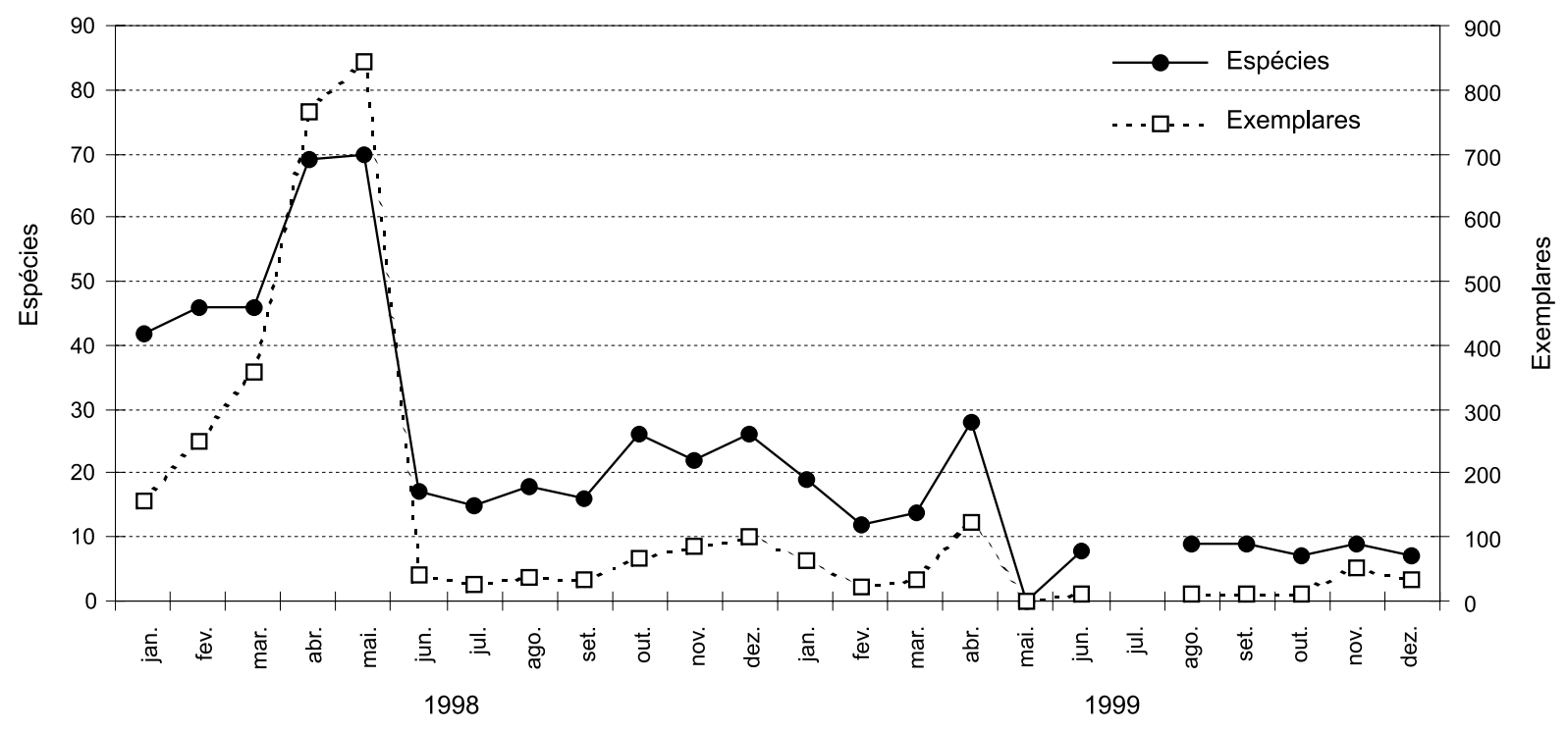

Fig. 2. Número de espécies e exemplares de Arctiinae coletados com armadilha luminosa, mensalmente, em Camaquã. Período 1998 e 1999.

por um exemplar ("singletons") e 17 por dois ("doubletons"); a quantidade destas para cada local encontra-se na Tabela V. Já com mais de 100 exemplares, ocorreram 21, sendo que somente Cosmosoma centrale (Walker) e Leucanopsis leucanina (R. Felder \& Rogenhofer) foram registradas em todos os locais; com mais de 1.000 exemplares aparece somente Philoros affinis (Rothschild) que também ocorreu em todos os locais. Esta relação de muitas espécies com poucos indivíduos, também foi encontrada por SPECHT (2001) para os noctuídeos no mesmo conjunto de amostragem. Isto é coerente com o enfatizado por CAMARGo (1999), analisando comunidades de lepidópteros no Cerrado, que comenta ser uma tendência para as comunidades tropicais. Também pode ser evidenciado, pelo número elevado de espécies acidentais e acessórias, pela classificação de Bodenheimer, em ambos os anos para todos os locais (Tabela I).

Em 1998 (Tabela I), Camaquã foi o local com maior número de exemplares coletados $(35,6 \%)$, seguido de São Pedro da Serra $(28,2 \%)$ e Iraí $(21,2 \%)$. Quando comparadas as médias cujos valores foram transformados em logaritmos (Tabela II), não houve diferença significativa entre elas, e estes três locais juntos correspondem a 85,0\% do total de Arctiinae coletados. O local com menor captura foi Mostardas com somente 1\% dos exemplares (local com menor número de amostras).

Com relação aos períodos de abundância para 1998, em Camaquã a maior abundância ocorreu em abril-maio (Fig. 2), porém, quando analisados estatisticamente (Tabela II), não há diferença entre estes e o mês de março; para Iraí, ocorreram

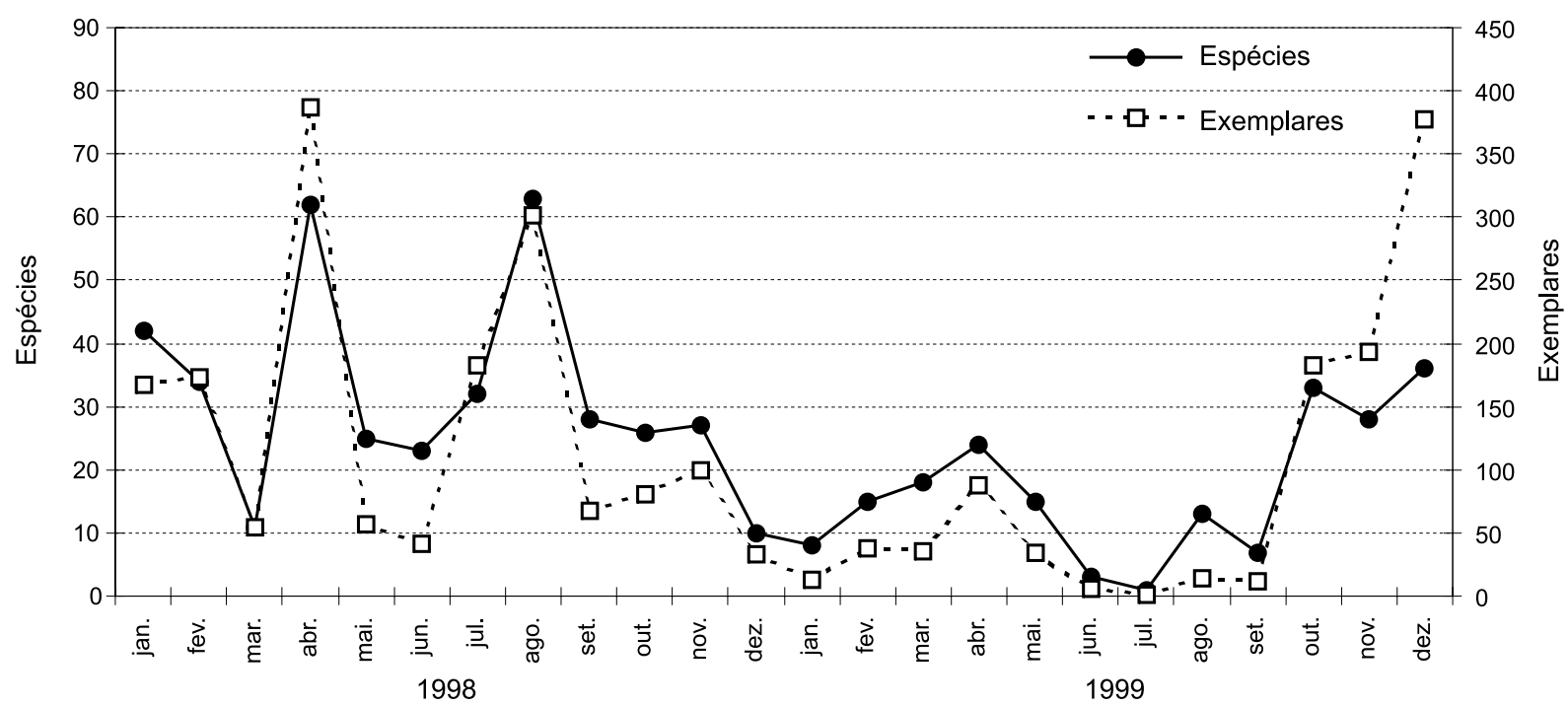

Fig. 3. Número de espécies e exemplares de Arctiinae coletados com armadilha luminosa, mensalmente, em Iraí. Período 1998 e 1999. 


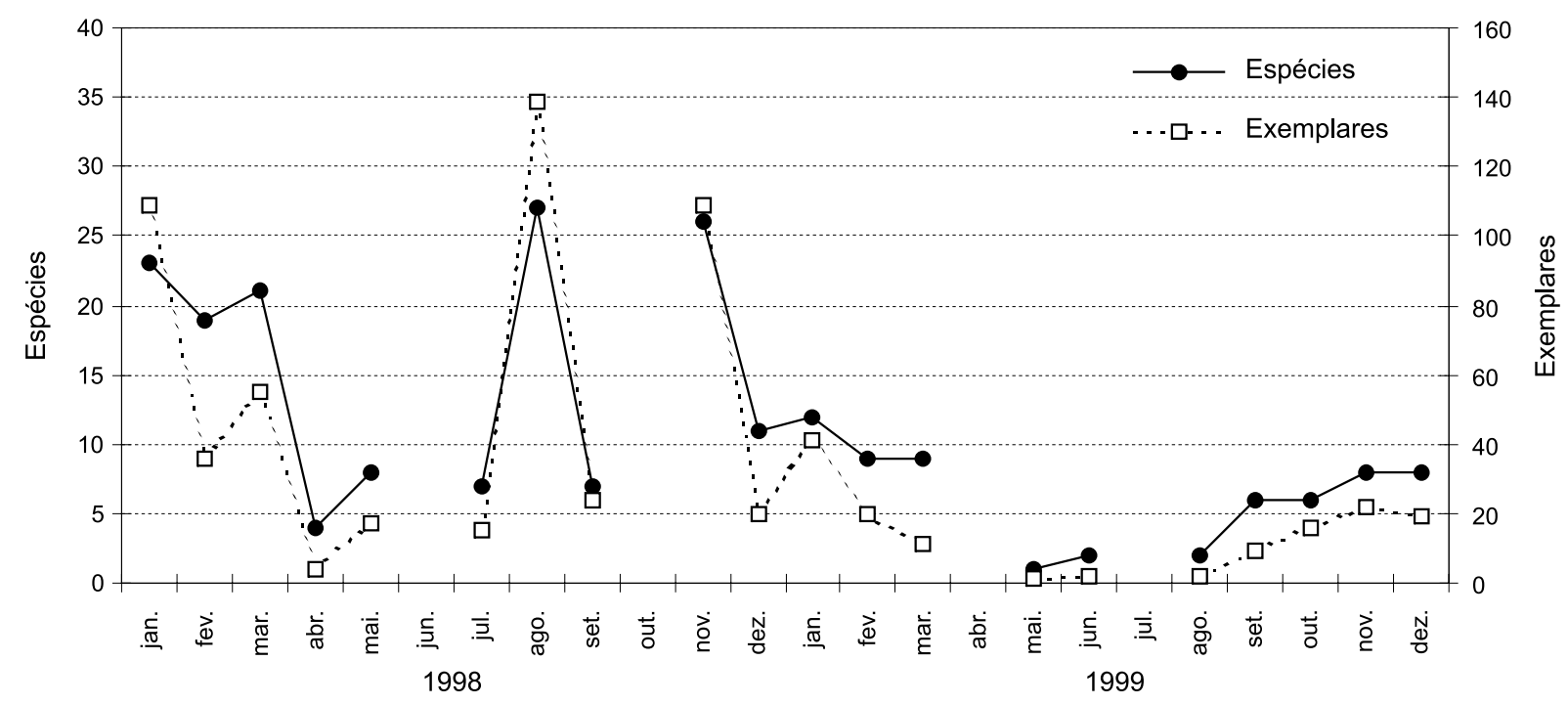

Fig. 4. Número de espécies e exemplares de Arctiinae coletados com armadilha luminosa, mensalmente, em Lagoa Vermelha. Período 1998 e 1999.

dois períodos de maior abundância, um em abril e outro em agosto (Fig. 3), porém na análise (Tabela II) aparecem sem diferença com os meses de janeiro, fevereiro e julho; Lagoa Vermelha teve abundâncias altas em janeiro, agosto e novembro (Fig. 4), e estatisticamente (Tabela II) estes não foram diferentes a março; Piratini teve alta abundância em fevereiro-março (Fig. 6), significativamente diferentes (Tabela II) dos demais meses; São Pedro da Serra apresentou abundâncias maiores em janeiro, março-abril, agosto e dezembro (Fig. 7), entretanto estatisticamente (Tabela II) não diferiram de fevereiro e novembro; e Mostardas é impróprio fazer algum destaque (Fig. 5), pois não houve diferença estatística entre os meses (Tabela II), devido ao número reduzido de exemplares.
Na Fig. 8, onde estão representados os totais para os seis locais, notam-se para 1998, maiores abundâncias nos meses de verão até início de outono e também no mês de agosto, não acompanhando o esperado pela sazonalidade. A linha de tendência teve coeficiente de determinação baixo tanto para indivíduos $\left(r^{2}=0,532\right)$, quanto para espécies $\left(r^{2}=0,327\right)$ e, por isso, a linha de tendência não foi incorporada na figura para 1998, onde se espera que num mês de inverno ocorra um número menor de espécies e indivíduos. A análise estatística (Tabela II) evidencia não haver diferença significativa entre os valores de janeiro a abril e agosto. Apesar do mês de maio apresentar um total elevado, a grande variação do número de exemplares, tanto nas armadilhas como nos locais, ocasionou sua exclusão no agrupamento dos meses de maior abundância.

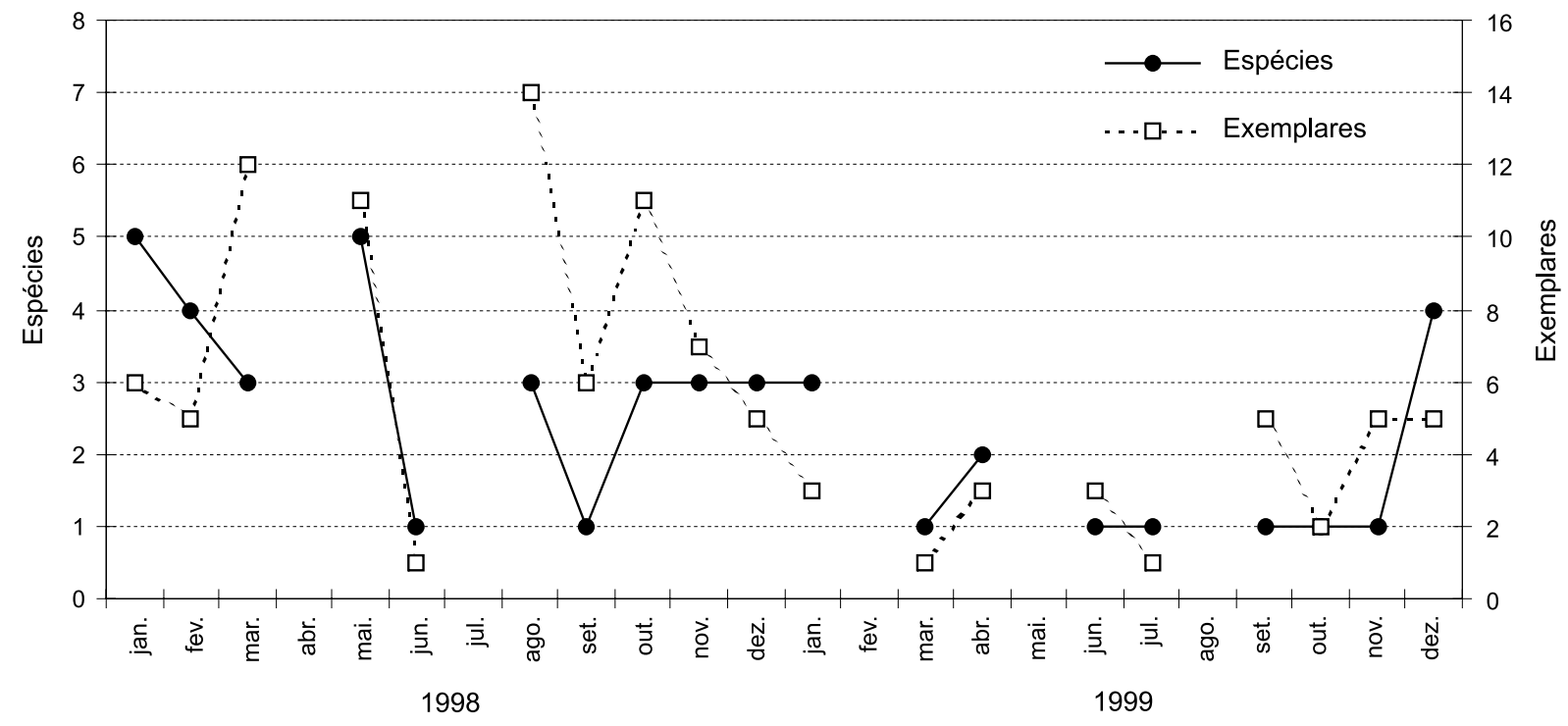

Fig. 5. Número de espécies e exemplares de Arctiinae coletados com armadilha luminosa, mensalmente, em Mostardas. Período 1998 e 1999. 
Tabela II. Números médios mensais de exemplares de Arctiinae coletados com armadilha luminosa, em municípios do Rio Grande do Sul, nos anos de 1998 e 1999. Valores transformados em logaritmo base 10 de $(\mathrm{x}+1)$.

\begin{tabular}{|c|c|c|c|c|c|c|c|c|c|c|c|c|c|c|}
\hline & & Camaquã & & Iraí & & Lagoa Ve & ielha & Mostar & das & Piratin & & São Pedro da & Serra & Total \\
\hline & neiro & 1,8 & $\mathrm{AB}$ & $\mathrm{cd}$ & & & $F$ & & $\mathrm{C}$ & $3 \mathrm{bc}$ & B & b & A & $+\mathrm{al}$ \\
\hline & Fevereiro & 2,09 & A & 1,939 & A & & B & & $\mathrm{C}$ & $964 \mathrm{ab}$ & A & & A & \\
\hline & Março & $2,256 \mathrm{ab}$ & A & cde & A & & $\mathrm{AB}$ & $557 \mathrm{a}$ & $\mathrm{B}$ & $2,038 \mathrm{a}$ & A & 2 & A & \\
\hline & Abril & 2,56 & A & $2,275 \mathrm{a}$ & A & $0,451 \mathrm{~d}$ & $\mathrm{~B}$ & & & $0,477 \mathrm{~d}$ & B & $2 a b$ & A & \\
\hline 1 & Maio & $2,617 \mathrm{a}$ & A & 1,462 cde & B & $0,972 \mathrm{~cd}$ & $\mathrm{BC}$ & $9 \mathrm{a}$ & $\mathrm{CD}$ & $0,150 \mathrm{~d}$ & $\mathrm{D}$ & & $\mathrm{BC}$ & $1 \mathrm{~cd}$ \\
\hline 9 & Junho & $1,243 \mathrm{ef}$ & A & $1,319 \mathrm{de}$ & A & & & & B & 9 d & B & $0 \mathrm{~d}$ & A & 0,790 e \\
\hline 9 & Julho & & $\mathrm{AB}$ & 1,83 & A & $0,929 \mathrm{~cd}$ & $\mathrm{~B}$ & & & & & & $\mathrm{AB}$ & 3 cd \\
\hline 8 & Agost & $1,296 \mathrm{def}$ & $\mathrm{BC}$ & 2,13 & A & $1,847 \mathrm{a}$ & $\mathrm{AB}$ & $0,795 \mathrm{a}$ & $\mathrm{C}$ & & & 2,2 & A & $1,656 \mathrm{ab}$ \\
\hline 8 & Setembro & $1,259 \mathrm{ef}$ & A & 1,46 & A & $0,972 \mathrm{~cd}$ & A & $0,602 \mathrm{a}$ & A & & & & $\mathrm{A}$ & 1,0 \\
\hline & Outubro & cdef & A & 1,6 & $\mathrm{~A}$ & & & $0,812 \mathrm{a}$ & $\mathrm{B}$ & $1,057 \mathrm{c}$ & $\mathrm{B}$ & & $\mathrm{B}$ & 9 cd \\
\hline & Novembro & 1,638 cde & A & 1,6 & A & 1,6 & A & $1 \mathrm{a}$ & B & $7 \mathrm{c}$ & $\mathrm{AB}$ & bcd & A & 8 bc \\
\hline & Dezem & 1,66 & A & $1,2 ?$ & $\mathrm{AB}$ & & $\mathrm{AB}$ & & B & & B & $\mathrm{cd}$ & A & 4 cd \\
\hline & Total & 1,769 & $\mathbf{A}$ & 1,689 & $\mathbf{A}$ & 1,233 & B & $\mathbf{0 , 5 5 7}$ & D & 0,998 & C & 1,677 & $\mathbf{A}$ & \\
\hline & & & & & & & & & $\mathrm{C}$ & & & & ${ }^{2}+2+2$ & 8 a \\
\hline & Fevereir & & $\mathrm{AB}$ & & $\mathrm{AB}$ & & B & & & & $\mathrm{C}$ & & A & 0 a \\
\hline & Março & 1,2 & A & & A & $0,778 \mathrm{abcd}$ & A & & B & 0,1 & B & 1, & $\mathrm{~A}$ & 18 a \\
\hline & Abril & 1,7 & A & 1,6 & $\mathrm{AB}$ & & & $0,389 \mathrm{a}$ & $\mathrm{C}$ & $0,239 \mathrm{c}$ & $\mathrm{C}$ & 1, & B & 1,005 a \\
\hline $\begin{array}{l}1 \\
9\end{array}$ & Maio & $0,000 \mathrm{e}$ & $\mathrm{C}$ & $1,254 \mathrm{bcd}$ & A & & $\mathrm{C}$ & & & $0,239 \mathrm{c}$ & $\mathrm{C}$ & $0,739 a b c$ & B & 0,476 b \\
\hline 0 & Junho & $0,812 \mathrm{~cd}$ & A & $0,422 \mathrm{de}$ & A & $0,239 \mathrm{~cd}$ & A & 0,501 a & A & $0,150 \mathrm{c}$ & A & & & 0,385 bc \\
\hline 9 & Julho & & & $0,150 \mathrm{e}$ & A & & & $0,150 \mathrm{a}$ & A & $0,422 \mathrm{bc}$ & A & 0, & A & 0,181 c \\
\hline 9 & Agosto & & A & $0,841 \mathrm{~cd}$ & A & & A & & & $0,540 \mathrm{bc}$ & A & $0,451 \mathrm{bc}$ & A & 0,531 b \\
\hline & Setembro & & $\mathrm{AB}$ & & A & abcd & $\mathrm{AB}$ & & B & & A & & A & 0,906 a \\
\hline & Outubro & $0,866 \mathrm{bcd}$ & $\mathrm{BC}$ & 1,7 & A & $a b$ & $\mathrm{BC}$ & $39 a$ & $\mathrm{C}$ & $1,322 \mathrm{a}$ & $\mathrm{AB}$ & $0,977 \mathrm{ab}$ & $\mathrm{ABC}$ & 1,023 a \\
\hline & Novembro & $1,417 \mathrm{abc}$ & $\mathrm{AB}$ & 1,98 & $\mathrm{~A}$ & $1,054 \mathrm{a}$ & $\mathrm{AB}$ & $0,540 \mathrm{a}$ & B & $0,500 \mathrm{bc}$ & B & $0,739 a b c$ & $\mathrm{~B}$ & 1,039 a \\
\hline & Dezembro & $1,211 \mathrm{abc}$ & B & & A & $0,866 a b c$ & $\mathrm{BC}$ & $0,540 \mathrm{a}$ & $\mathrm{BC}$ & $0,239 \mathrm{c}$ & $\mathrm{C}$ & $1,078 \mathrm{ab}$ & B & 1,027 a \\
\hline & Total & 1,014 & $\mathbf{A B}$ & 1,190 & A & $\mathbf{0 , 7 3 7}$ & C & 0,343 & D & 0,528 & D & $\mathbf{0 , 9 7 7}$ & B & \\
\hline
\end{tabular}

* Médias seguidas por letras distintas diferem entre si pelo teste de Duncan ao nível de 5\%; minúsculas, em cada coluna, referem-se às diferenças entre meses e maiúsculas, em cada linha, entre locais.

Em 1999 (Tabela I), Iraí foi o local com maior número de exemplares coletados (49,2\%), seguido de Camaquã $(18,2 \%)$ e São Pedro da Serra (17,6\%); quando comparados estatisticamente (Tabela II) não houve diferença entre Iraí e Camaquã e, como no ano anterior, estes três locais tiveram a maior abundância com $85,0 \%$ dos exemplares. Novamente, o local com menor captura foi Mostardas com apenas 1,4\% do total.

Com relação aos períodos de abundância de Arctiinae para 1999, Camaquã teve maior abundância em abril (Fig. 2), porém estatisticamente (Tabela II) não há diferença entre este e janeiro, março, novembro e dezembro; para Iraí, dezembro foi de maior abundância (Fig. 3), porém na análise estatística (Tabela II) aparece sem diferença entre os meses de abril, outubro e novembro; Lagoa Vermelha teve alta abundância em janeiro (Fig. 4), e segundo a análise (Tabela II) não foi diferente a fevereiro-março e setembro-dezembro; Piratini teve período de abundância alta em setembro-outubro (Fig. 6), estatisticamente (Tabela II) igual a janeiro; São Pedro da Serra com maior abundância em janeiro-fevereiro (Fig. 7) porém significativamente diferentes (Tabela II) somente de julho e agosto; e Mostardas é inconveniente fazer algum destaque (Fig. 5), pois não houve diferença estatística entre os meses
(Tabela II), devido ao número reduzido de exemplares.

Quando observada a abundância em 1999 (Fig. 8), para o total dos seis locais, nota-se dezembro como o de maior abundância, entretanto na análise estatística (Tabela II) só há diferença com relação aos meses de maio a agosto ou seja, fim de outono e inverno. Nota-se (Fig. 8) o acompanhamento da sazonalidade pois a linha de tendência demonstra esta expectativa biológica, seu coeficiente de determinação para exemplares $\left(\mathrm{r}^{2}=0,857\right)$ foi alto e também para espécies $\left(\mathrm{r}^{2}=\right.$ $0,536)$.

A análise de regressão com os fatores climáticos, não permitiu evidenciar influência significativa na ação dos mesmos nos dias das coletas. O fato de Arctiinae não apresentar estreito relacionamento com os fatores climáticos quanto à flutuação do número de espécies e exemplares, foi evidenciado por FERREIra et al. (1995), que admitem outros fatores bióticos e abióticos, não observados, influenciando na abundância e riqueza de espécies.

Os maiores valores de riqueza de espécie em ambos os anos, foram encontradas em Iraí, Camaquã e São Pedro da Serra. Em relação às espécies exclusivas, Iraí foi superior nos dois anos, seguido por Camaquã (Tabela III).

Os valores obtidos para os índices de Shannon $\left(H^{\prime}\right)$ (Tabela 


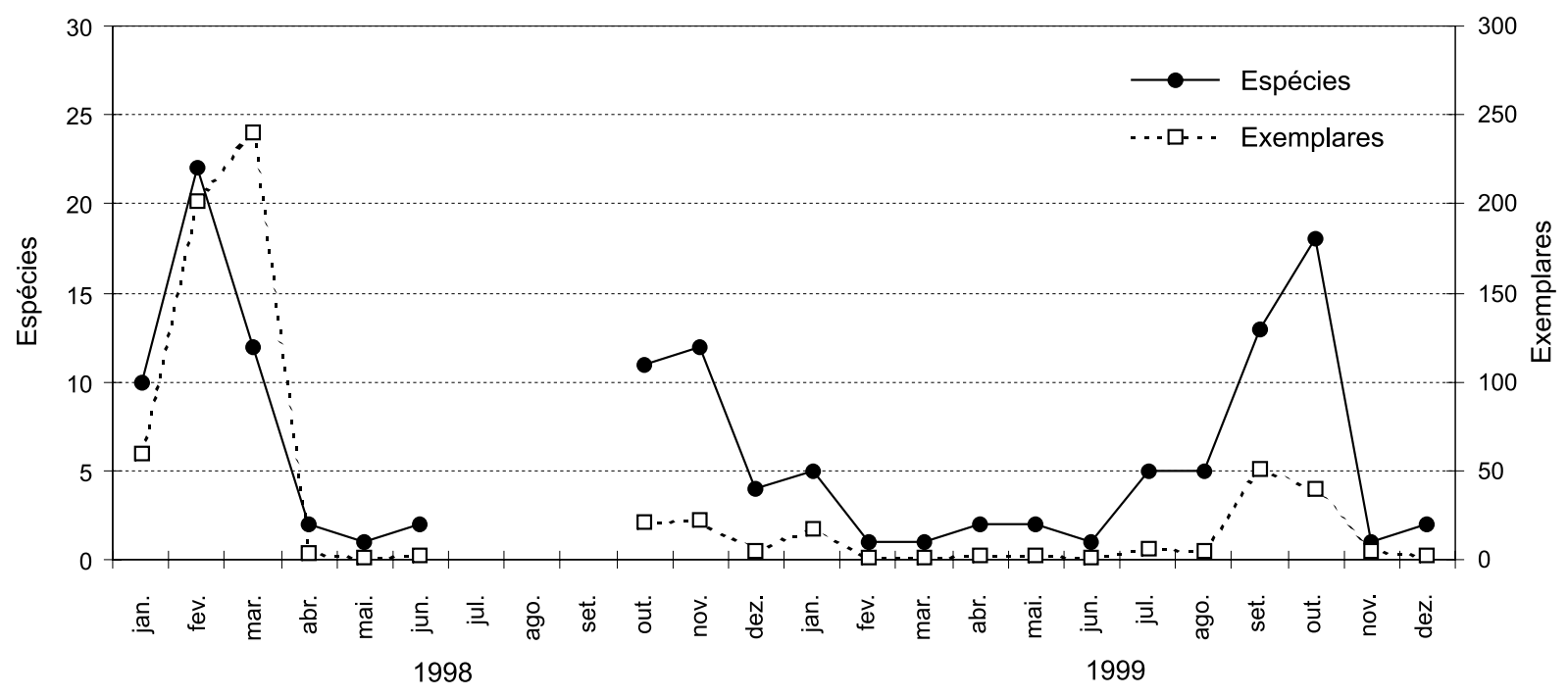

Fig. 6. Número de espécies e exemplares de Arctiinae coletados com armadilha luminosa, mensalmente, em Piratini. Período 1998 e 1999.

III), em 1998 variaram de 5,04 em São Pedro da Serra a 2,16 em Piratini; já em 1999 variaram de 4,40 em São Pedro da Serra a 1,90 em Mostardas. Ainda, em relação ao índice de diversidade de Shannon, a comparação pelo teste $t$ de Student entre os anos para cada local, somente Lagoa Vermelha e Mostardas não apresentaram diferenças significativas. Outro fato a evidenciar é de que para a maioria dos locais os valores em 1998 foram superiores aos de 1999, exceto para Piratini que devido à inexistência de dados entre julho a setembro no primeiro ano influenciou o seu baixo índice de diversidade.

Na Tabela IV, encontram-se os valores do teste " $t$ " para os índices de diversidade Shannon $\left(H^{\prime}\right)$ entre os seis municípios em cada ano. Em 1998, houve diferença estatística significativa entre: Camaquã e Lagoa Vermelha; Lagoa Vermelha e São Pedro da Serra; Mostardas e Piratini com os demais locais, mas não entre eles. Estas diferenças são evidenciadas pelas desigualdades dos valores de abundância e riqueza de espécies apresentados entre os locais. Em 1999, somente houve diferença estatística entre Mostardas e os demais, isto também é devido aos baixos valores de abundância e riqueza de espécies encontrados neste local.

Quanto ao índice de Brillouin (H) (Tabela III), em 1998, variou de 4,92 em São Pedro da Serra a 2,01 em Piratini e, em 1999, variou de 4,10 em São Pedro da Serra a 1,54 em Mostardas.

Apesar dos índices terem pressupostos diferentes, o de Shannon, mais comumente utilizado, assume que os indivíduos são uma amostra da população, obtidos aleatoriamente e o de Brillouin, que é considerado o melhor para indicar a diversidade

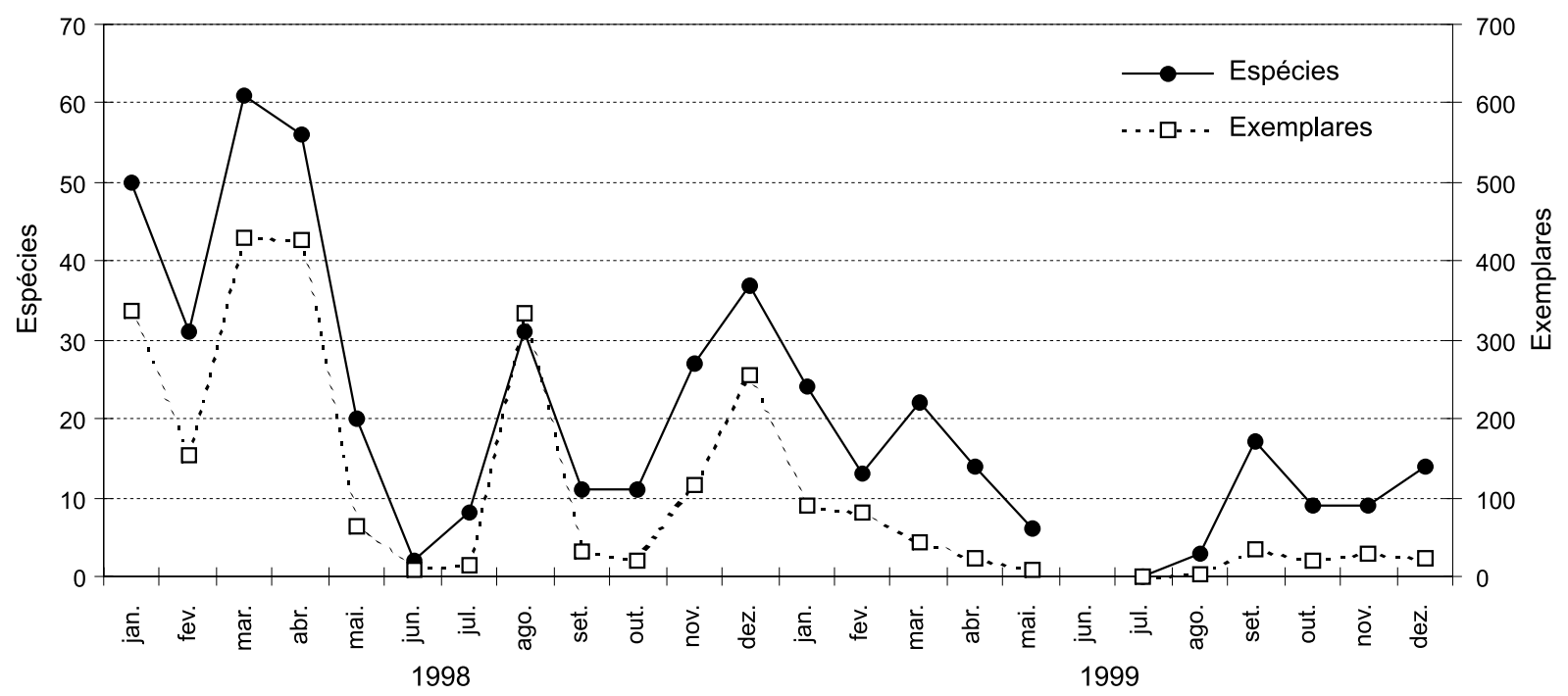

Fig. 7. Número de espécies e exemplares de Arctiinae coletados com armadilha luminosa, mensalmente, em São Pedro da Serra. Período 1998 e 1999. 


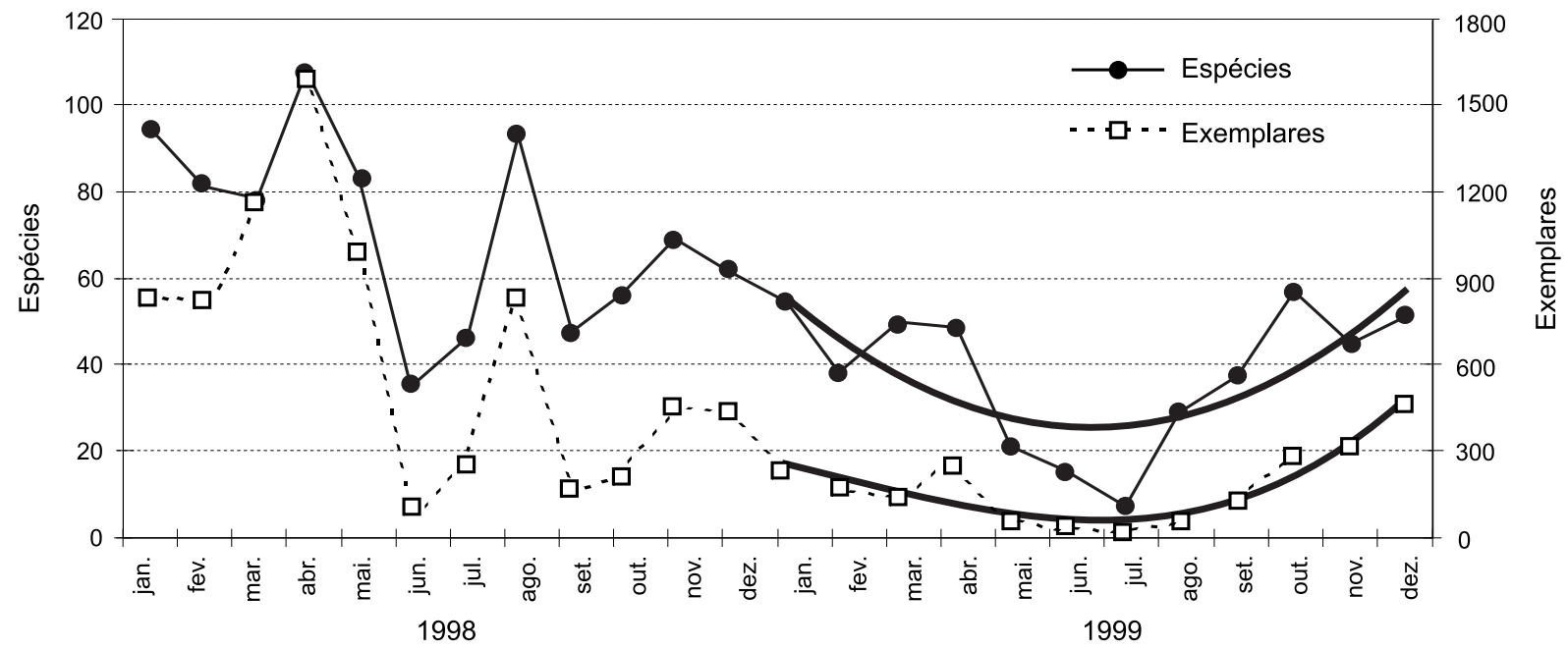

Fig. 8. Totais de espécies e exemplares de Arctiinae coletados com armadilha luminosa, mensalmente, em seis locais do Rio Grande do Sul. Período 1998 e 1999. Com linhas de tendência para 1999.

de insetos coletados com armadilha luminosa, assume que os indivíduos coletados são a totalidade da população e seus valores devem ser tratados como diferentes significativamente entre si (MAgurRan 1988); os dois índices (Tabela III) apontaram os mesmos locais de maior e menor diversidade em cada ano.

Os maiores valores de diversidade obtidos para os índices de Shannon e Brillouin, comparados aos encontrados por MARINONi \& Dutra (1996) no Paraná, com os devidos ajustes, para os atuais Ctenuchini e Euchromiini, foram levemente superiores em 1998 e praticamente iguais em 1999. Foram porém, inferiores aos valores de diversidade encontrados para os noctuídeos amostrados nos mesmos locais por SPECHT (2001).

O índice de uniformidade de Shannon ( $E^{\prime}$ ) (Tabela III) em 1998, variou de 0,770 em Lagoa Vermelha a 0,403 em Piratini, e em 1999 variou de 0,854 em Piratini a 0,657 em Iraí; o de Brillouin (E) em 1998, variou de 0,774 em São Pedro da Serra a 0,395 em Piratini e em 1999 variou de 0,902 em Piratini a 0,657 em Iraí. Observando a Tabela III, nota-se que em 1998 houve uma pequena diferença entre os locais quanto aos índices de uniformidade, porém os valores são muito semelhantes, quase não havendo diferença entre Lagoa Vermelha e São Pedro da Serra; quanto a 1999 os dois índices apontaram para o mesmo local, sendo Piratini o de maior uniformidade entre as espécies.

Igualmente ao constatado por MARINONI \& DUTRA (1996), os valores de abundância e riqueza de espécies não foram fundamentais na definição dos maiores índices de uniformidade. Tanto Lagoa Vermelha (uniformidade de Shannon) quanto São Pedro da Serra (uniformidade de Brillouin), em 1998, não foram os locais com os maiores valores de abundância e riqueza de espécies; o mesmo também ocorreu para Piratini (uniformidade de Shannon e Brillouin) em 1999. Já os locais com os menores índices de uniformidade (Tabela III) sofreram forte dominância de algumas poucas espécies sobre as demais (Tabela I); em 1998, Piratini teve Philoros affinis
(Rothschild), com 394 exemplares de um total de 558 e, em 1999, Iraí teve Cosmosoma centrale (Walker), Dycladia lucetius (Stoll) e Philoros affinis (Rothschild), com 538 de um total de 997 exemplares.

Nos dois anos ocorreram somente seis espécies em todos os locais, Cosmosoma centrale (Walker), Dysschema sacrifica (Hübner), Idalus agastus Dyar, Leucanopsis leucanina (R. Felder \& Rogenhofer), Philoros affinis (Rothschild) e Symphlebia catenata (Schaus) e 71 espécies exclusivas (Tabelas I e III), sendo que Iraí com 28 delas, foi o local com a maior representatividade.

Os estimadores de riqueza (Tabela V) forneceram diferentes valores sendo que, em Camaquã, o procedimento "Jackknife 2" calculou que podem ser encontradas 149 espécies (mais $34,3 \%$ ); a estimativa de mais baixo valor foi o "Bootstrap" com 122 espécies. Em Iraí o procedimento "Jackknife 2" calculou que podem ser encontradas 135 espécies (mais 18,4\%); a estimativa de mais baixo valor foi o "Chao 2" com 123 espécies. Em Lagoa Vermelha, o procedimento "Chao 1" calculou que podem ser encontradas 126 espécies (mais 75,4\%); a estimativa de mais baixo valor foi o "Bootstrap" com 84 espécies. Em Mostardas, o procedimento "Michaelis-Mentem" calculou que podem ser encontradas 20 espécies (mais 47,1\%); a estimativa de mais baixo valor foi o "Chao 1" com 15 espécies. Em Piratini, o procedimento "Michaelis-Mentem" calculou que podem ser encontradas 88 espécies (mais 66,2\%); a estimativa de mais baixo valor foi o "Bootstrap" com 62 espécies. Em São Pedro da Serra, o procedimento "Jackknife 2" calculou que podem ser encontradas 150 espécies (mais 43,7\%); a estimativa de mais baixo valor foi o "Bootstrap" com 118 espécies.

Os maiores valores de abundância, riqueza de espécies e estimativas de riqueza foram obtidos em Camaquã, Iraí, Lagoa Vermelha e São Pedro da Serra, locais com ambientes florestais típicos bem conservados. Specht (2001) também evidenciou que estes locais foram os de maiores valores para Noctuidae. Coerente com a afirmação de Cook \& Graham (1996), que os 
Tabela III. Número de espécies (S) e de exemplares $(\mathrm{N})$; valores dos índices de diversidade de Shannon $\left(H^{\prime}\right)$ e Brillouin $(H)$, uniformidade de Shannon $\left(E^{\prime}\right)$ e Brillouin $(E)$, número de espécies exclusivas para cada local e ano (sp. excl.) e para os dois anos (sp. excl. total) e teste $t$ comparando o $H$ ' entre os anos para cada local para os Arctiinae capturados com armadilha luminosa em municípios do Rio Grande do Sul nos anos de 1998 e 1999.

\begin{tabular}{|c|c|c|c|c|c|c|c|}
\hline & & Camaquã & Iraí & Lagoa Vermelha & Mostardas & Piratini & São Pedro da Serra \\
\hline & $\mathrm{S}$ & 107 & 109 & 59 & 14 & 41 & 95 \\
\hline 1 & $\mathrm{~N}$ & 2768 & 1649 & 528 & 78 & 558 & 2192 \\
\hline 9 & $H^{\prime}(\log 2)$ & 5,01 & 4,92 & 4,53 & 2,57 & 2,16 & 5,04 \\
\hline 9 & $H(\log 2)$ & 4,91 & 4,76 & 4,28 & 2,26 & 2,01 & 4,92 \\
\hline \multirow[t]{4}{*}{8} & $E^{\prime}$ & 0,744 & 0,728 & 0,770 & 0,675 & 0,403 & 0,766 \\
\hline & $E$ & 0,744 & 0,736 & 0,771 & 0,668 & 0,395 & 0,774 \\
\hline & sp. excl. & 13 & 31 & 6 & 1 & 4 & 12 \\
\hline & $\mathrm{S}$ & 62 & 72 & 36 & 7 & 31 & 54 \\
\hline 1 & $\mathrm{~N}$ & 369 & 997 & 143 & 28 & 134 & 356 \\
\hline 9 & $H^{\prime}(\log 2)$ & 3,99 & 4,05 & 4,01 & 1,90 & 4,23 & 4,40 \\
\hline 9 & $H(\log 2)$ & 3,66 & 3,89 & 3,56 & 1,54 & 3,79 & 4,10 \\
\hline \multirow[t]{3}{*}{9} & $E^{\prime}$ & 0,670 & 0,657 & 0,776 & 0,676 & 0,854 & 0,765 \\
\hline & $E$ & 0,664 & 0,657 & 0,773 & 0,772 & 0,902 & 0,778 \\
\hline & sp. excl. & 13 & 21 & 11 & 1 & 8 & 5 \\
\hline \multirow{2}{*}{\multicolumn{2}{|c|}{$\begin{array}{l}\text { sp. excl. total } \\
\text { Teste t }\left(H^{\prime}\right)\end{array}$}} & 14 & 28 & 10 & 1 & 9 & 9 \\
\hline & & $4,20^{(*)}$ & $4,83^{(*)}$ & $1,36^{(\mathrm{ns})}$ & $1,40^{(\mathrm{ns})}$ & $5,7^{(*)}$ & $2,53^{(*)}$ \\
\hline
\end{tabular}

${ }^{(\mathrm{ns})}=$ não significativo ao nível de $5 \% ;^{(*)}=$ significativo ao nível de $5 \%$.

ambientes florestais são os que apresentam melhores índices de riqueza de espécies para os lepidópteros.

\section{CONCLUSÕES}

O presente estudo permitiu constatar que ocorreu uma grande variação quanto à abundância, sendo que em 1998 foi muito superior a 1999, o mesmo ocorrendo também quanto a riqueza de espécies. Detectou-se que $47 \%$ das espécies ocorreram em frequiências muito baixas, com até 10 exemplares. Camaquã, Iraí e São Pedro da Serra foram os locais com maior abundância e riqueza de espécies.

Espécies dos gêneros Correbidia, Ochrodota e Sphecosoma são registradas pela primeira vez para o Rio Grande do Sul.
Evidenciou-se que em 1998, por ter sido atípico, os dados não permitiram conveniente adequação para uma linha de tendência, que ao contrário, em 1999, mostraram-se harmônicos à sazonalidade.

Os fatores climáticos estudados, com observações nos dias das coletas, não permitiram explicar as variações quanto a abundância e riqueza de espécies, que poderiam ser atribuídas às características próprias de cada local, em especial ao tipo de vegetação e seu estado de conservação, bem como microclimas.

Os maiores índices de diversidade, em 1998, foram encontrados em Camaquã, Iraí e São Pedro da Serra; já em 1999, em Iraí, Piratini e São Pedro da Serra, estes locais apresentam suas vegetações típicas em bom estado de conservação.

Segundo as estimativas de riqueza, há expectativa de serem

Tabela IV. Valores do teste " $t$ " comparando os índices de diversidade de Shannon $(H$ ') para Arctiinae capturados com armadilha luminosa em municípios do Rio Grande do Sul, entre os locais, nos anos de 1998 e 1999 (negrito).

\begin{tabular}{|c|c|c|c|c|c|c|}
\hline & Camaquã & Iraí & Lagoa Vermelha & Mostardas & Piratini & São Pedro da Serra \\
\hline Camaquã & - & $0,25^{(n s)}$ & $0,06^{\text {(ns) }}$ & $3,81^{(*)}$ & $0,6^{(n s)}$ & 1,29 (ns) \\
\hline Iraí & $0,59^{(\mathrm{ns})}$ & - & $0,12^{(n s)}$ & $4,55^{(*)}$ & $0,55^{(n s)}$ & $0,966^{(n s)}$ \\
\hline Lagoa Vermelha & $2,28^{(*)}$ & $1,75^{(\mathrm{ns})}$ & - & $3,25^{(*)}$ & $0,47^{(n s)}$ & $0,98^{(n s)}$ \\
\hline Mostardas & $8,02^{(*)}$ & $7,64^{(*)}$ & $6,23^{(*)}$ & - & $3,69^{(*)}$ & $4,899^{*}$ \\
\hline Piratini & $17,49^{(*)}$ & $6,98^{(*)}$ & $10,17^{(*)}$ & $0,65^{(\mathrm{ns})}$ & - & $0,42^{(n s)}$ \\
\hline São Pedro da Serra & $0,15^{(\mathrm{ns})}$ & $0,61^{(\mathrm{ns})}$ & $2,34^{(*)}$ & $8,03^{(*)}$ & $17,02^{(*)}$ & - \\
\hline
\end{tabular}

(ns) = não significativo ao nível de $5 \%{ }^{(*)}=$ significativo ao nível de $5 \%$. 
Tabela V. Estimativas para o total de Arctiinae. Número de amostras, espécies, número de espécies representadas por apenas um ("Singletons"), dois indivíduos ("Doubletons") e riqueza de espécies, segundo vários estimadores, referentes aos Arctiinae capturados com armadilha luminosa em municípios do Rio Grande do Sul nos anos de 1998 e 1999.

\begin{tabular}{lcccccc}
\hline & Camaquã & Iraí & Lagoa Vermelha & Mostardas & Piratini & São Pedro da Serra \\
\hline Amostras & 46 & 48 & 40 & 38 & 42 & 44 \\
Espécies & 111 & 114 & 72 & 14 & 53 & 105 \\
"Singletons" & 25 & 19 & 28 & 4 & 15 & 27 \\
"Doubletons" & 8 & 14 & 6 & 4 & 12 & 11 \\
"Chao 1" & 144,5 & 125,4 & 126,3 & 15,3 & 66,1 & 134,3 \\
"Chao 2" & 134,3 & 123,6 & 112,7 & 16,1 & 74,1 & 134,1 \\
"Bootstrap" & 122,7 & 124,9 & 84,3 & 16,3 & 62,6 & 118,8 \\
"Jackknife 1" & 136,4 & 134,6 & 100,3 & 18,9 & 74,5 & 135,0 \\
"Jackknife 2" & 149,1 & 135,0 & 119,5 & 19,9 & 86,1 & 150,9 \\
"Michaelis-Mentem" & 124,9 & 132,1 & 109,6 & 20,6 & 88,1 & 119,0 \\
\hline
\end{tabular}

encontradas mais 34\% de espécies em Camaquã, 18\% em Iraí, 75\% em Lagoa Vermelha, 47\% em Mostardas, 66\% em Piratini e $43 \%$ em São Pedro da Serra. Conseqüentemente, o inventariamento de Arctiinae para estes locais, deve ser ampliado no tempo e no número de pontos de coleta.

Agradecimentos. Ao colega Alexandre Specht, pela grande ajuda e doação de todo o material entomológico de seu estudo, sem o qual este trabalho não poderia ser efetuado e a Hervé de Toulgoët, do Muséum National d'Histoire Naturelle, Paris, pelo auxílio na identificação de algumas espécies.

\section{REFERÊNCIAS}

Arend, L. M. 1997. Geografia física, p. 22-53. In: G. R. Hoffmann; L. M. Arend; J. C. B da Silveira; H. R. Bellomo \& J. L. M. Nunes (Eds.). Rio Grande do Sul-Aspectos da geografia. $4^{\text {a }}$ ed. Porto Alegre, Martins Livreiro, 104 p.

Brzustowski, J. 1997. Krebs Ecological Methodology for Windows, version 0.9 .

Butler, L.; V. Kondo; E. M. Barrows \& E. C. Townsend. 1999. Effects of weather conditions and trap types on sampling for richness and abundance of forest macrolepidoptera. Environmental Entomology 28(5): 795-811.

Camargo, A. J. A. DE. 1999. Estudo comparativo sobre a composição e a diversidade de lepidópteros noturnos em cinco áreas da Região dos Cerrados. Revista Brasileira de Zoologia 16(2): 369-380.

Colwell, R. K. 2000. EstimateS: Statistical estimation of species richness and shared species from samples, version $6.0 \mathrm{~b} 1$.

Colwell, R. K. \& J. A. Coddington. 1994. Estimating terrestrial biodiversity through extrapolation. Philosophical Transaction of the Royal Society of London, Series B, 345: 101-118.

Cook, L. M. \& C. S. Graham. 1996. Evenness and species number in some moth populations. Biological Journal of the Linnean Society 58(1): 75-84.

Dorval, A.; J. C. Zanuncio; J. M. M. Pereira \& W. L. Gasperazzo. 1995. Análise faunística de Eupseudosoma aberrans Schaus, 1905 e Eupseudosoma involuta (Sepp, 1852) (Lepidoptera: Arctiidae) em Eucalyptus urophylla e Eucalyptus cleziana na região de Montes Claros, Minas Gerais. Revista Árvore 19(2): 228-240.

Ferreira, P. S. F.; A. S. Paula \& D. S. Martins. 1995. Análise faunística de Lepidoptera Arctiidae em área de reserva natural remanescente de floresta tropical em Viçosa, Minas Gerais. Anais da Sociedade Entomológica do Brasil 24(1): 123-133.

Frost, S. W. 1957. The Pennsylvania insect light trap. Journal of Economic Entomology 50(3): 287-292.

FrY, R. \& P. Waring. 1996. A guide to moth traps and their use. The Amateur Entomologist 24: 1-60.

Glantz, M. H. 1997. Currents of change: El Niño's impact on climate and society. Cambridge, Cambridge University Press, $194 \mathrm{p}$.

Hammond, P. C. \& J. C. Miller. 1998. Comparison of the biodiversity of Lepidoptera within three forested ecosystems. Annals of the Entomological Society of America 91(3): 323-328.

Hampson, G. F. 1898. Catalogue of the Lepidoptera Phalaenae in the British Museum. Volume 1. Catalogue of the Syntomidae in the collection of the British Museum. London, Taylor and Francis, 559 p.

HAYEK, L. A. C. \& M. A. BUZAS. 1997. Surveying natural populations. New York, Columbia University Press, 563 p.

Heppner, J. B. 1991. Faunal regions and the diversity of Lepidoptera. Tropical Lepidoptera 2(Suppl.1): 1-85.

Hilty, J. \& A. Merenlender. 2000. Faunal indicator taxa selection for monitoring ecosystem health. Biological Conservation 92: 185197.

Intachat, J. \& I. P. Woiwod. 1999. Trap design for monitoring moth biodiversity in tropical rainforests. Bulletin of Entomological Research 89: 153-163.

Jacobson, N. L. \& S. J. Weller. 2002. A cladistic study of the Arctiidae (Lepidoptera) by using characters of immatures and adults. Lanham, Thomas Say Publications in Entomology (Monograph)/ Entomological Society of America, 98 p.

Kitching, I. J. \& J. E. Rawlins. 1999. The Noctuoidea, p. 355-401. In: N. P. Kristensen (ed. vol.). Lepidoptera, Moths and Butterflies. Volume 1: Evolution, Systematics, and Biogeography. In: M. FIscher (ed.). Handbook of Zoology. Volume IV Arthropoda: Insecta. Berlin, de Gruyter, 491 p.

Kitching, R. L.; A. G. OrR; L. Thalib; H. Mitchell; M. S. Hopkins \& A. W. Graham. 2000. Moth assemblages as indicators of environmental quality in remnants of upland Australian rain forest. Journal of Applied Ecology 37: 284-297.

Krebs, C. J. 1999. Ecological Methodology. 2a ed. New York, Benjamin/ Cummings, $620 \mathrm{p}$.

Landau, B.; D. Prowell \& C. E. Carlton. 1999. Intensive versus longterm sampling to assess lepidopteran diversity in southern mixed mesophytic forest. Annals of the Entomological Society of America 92(3): 435-441. 
Lasmar, A. 1997. A fúria da natureza. Ecologia e Desenvolvimento 7(66): $16-19$

Leite, P. F. 2002. Contribuição ao conhecimento fitoecológico do Sul do Brasil. Ciência \& Ambiente 24: 51-73.

LÜBeck, G. M.; J. V. Oliveira \& R. P. Almeida. 1995. Análise faunística de lepidópteros coletados em duas comunidades agrícolas na Zona da Mata norte de Pernambuco. Anais da Sociedade Entomológica do Brasil 24(2): 353-370.

MagurRan, A. E. 1988. Ecological diversity and its measurement. Princeton, Princeton University Press, 179 p.

Marinoni, R. C. \& R. R. C. Dutra. 1996. Levantamento da fauna entomológica do Estado do Paraná. II. Ctenuchidae (Lepidoptera). Revista Brasileira de Zoologia 13(2): 435-461.

Oliver, I. \& A. J. BeatTiE. 1996. Designing a cost-effective invertebrate survey: a test of methods for rapid assessment of biodiversity. Ecological Applications 6(2): 594-607.

Pereira, J. M. M.; J. C. Zanuncio; J. H. Schoereder \& G. P. Santos. 1995. Agrupamento de oito povoamentos florestais em relação à fauna de lepidópteros daninhos ao eucalipto, através de análise de agrupamento. Revista Brasileira de Entomologia 39(3): 647652.

Quadros, F. L. F. DE \& V. DE P. Pillar. 2002. Transições floresta-campo no Rio Grande do Sul. Ciência \& Ambiente 24: 109-118.

Silveira Neto, S; O. Nakano; D. Barbin \& N. A. V. Nova. 1976. Manual de ecologia dos insetos. Piracicaba, Agronômica Ceres, 419 p.

SPECht, A. 2001. Diversidade e aspectos ecológicos dos noctuídeos
(Lepidoptera, Noctuidae) ocorrentes no Rio Grande do Sul Tese de Doutorado. Porto Alegre, Pontifícia Universidade Católica do Rio Grande do Sul, 71 p.

Teston, J. A. \& E. Corseuil. 2002. Arctiinae (Lepidoptera, Arctiidae) ocorrentes no Rio Grande do Sul, Brasil. Parte I. Pericopini. Biociências 10(2): 79-86.

Teston, J. A. \& E. Corseuil. 2003a. Arctiinae (Lepidoptera, Arctiidae) ocorrentes no Rio Grande do Sul, Brasil. Parte II. Arctiini, Callimorphini e Phaegopterini. Biociências 11(1): 69-80.

Teston, J. A. \& E. Conseuil. 2003b. Arctiinae (Lepidoptera, Arctiidae) ocorrentes no Rio Grande do Sul, Brasil. Parte III. Ctenuchini e Euchromiini. Biociências 11(1): 81-90.

Watson, A.; D. S. Fletcher \& I. W. B. Nye. 1995. Noctuoidea: Arctiidae, Cocytiidae, Ctenuchidae, Dilobidae, Dioptidae, Lymantriidae, Notodontidae, Strepsimanidae, Thaumetopoeidae \& Thyretidae. In: I. W. B. NyE (ed.). The generic names of moths of the world. Vol. 2. Reprinted. London, The Natural History Museum, 228 p.

Watson, A. \& D. T. Goodger. 1986. Catalogue of the Neotropical Tigermoths. Occasional Papers on Systematic Entomology 1: 171.

Weller, S. J.; R. B. Simmons; R. Boada \& W. E. Conner. 2000. Abdominal modifications occurring in wasp mimics of the CtenuchineEuchromiini clade (Lepidoptera: Arctiidae). Annals of the Entomological Society of America 93(4): 920-928. 